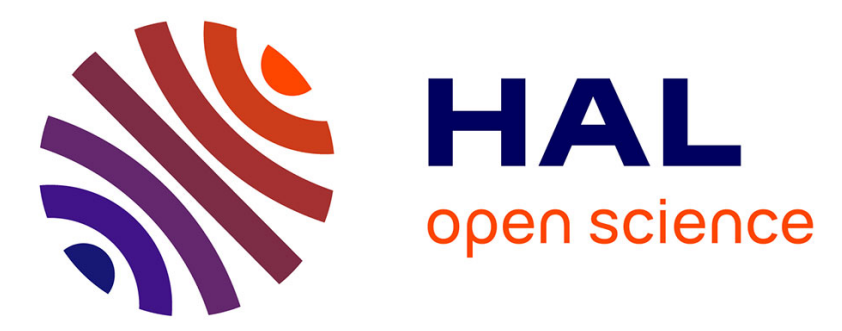

\title{
An Elementary Approach to Filtering in Systems with Fractional Brownian Observation Noise
}

\author{
M.L. Kleptsyna, A. Le Breton, M. C. Roubaud
}

\section{To cite this version:}

M.L. Kleptsyna, A. Le Breton, M. C. Roubaud. An Elementary Approach to Filtering in Systems with Fractional Brownian Observation Noise. RR-3439, INRIA. 1998. inria-00073251

\section{HAL Id: inria-00073251 \\ https://hal.inria.fr/inria-00073251}

Submitted on 24 May 2006

HAL is a multi-disciplinary open access archive for the deposit and dissemination of scientific research documents, whether they are published or not. The documents may come from teaching and research institutions in France or abroad, or from public or private research centers.
L'archive ouverte pluridisciplinaire $\mathbf{H A L}$, est destinée au dépôt et à la diffusion de documents scientifiques de niveau recherche, publiés ou non, émanant des établissements d'enseignement et de recherche français ou étrangers, des laboratoires publics ou privés. 


\title{
An elementary approach to filtering in systems with fractional Brownian observation noise
}

\author{
M.L. Kleptsyna, A. Le Breton and M. C. Roubaud
}

\section{$\mathbf{N}^{\circ} 3439$}

Juin 1998

THÈME 4 



\title{
An elementary approach to filtering in systems with fractional Brownian observation noise
}

\author{
M.L. Kleptsyna $^{*}$, A. Le Breton ${ }^{* *}$ and M. C. Roubaud ${ }^{* * *}$ \\ Thème 4 - Simulation et optimisation \\ de systèmes complexes \\ Projet SYSDYS
}

Rapport de recherche $\mathrm{n}^{\circ} 3439$ - Juin 1998 - 39 pages

\begin{abstract}
The problem of optimal filtering is addressed for a signal observed through a possibly nonlinear channel driven by a fractional Brownian motion. An elementary and completely self-contained approach is developed. An appropriate Girsanov type result is proved and a process - equivalent to the innovation process in the usual situation where the observation noise is a Brownian motion - is introduced. Zakai's approach is partly extended to derive filtering equations when the signal process is a diffusion. The case of conditionally Gaussian linear systems is analyzed. Closed form equations are derived both for the mean of the optimal filter and the conditional variance of the filtering error. The results are explicit in various special cases.
\end{abstract}

Key-words: Fractional Brownian motion, Girsanov formula, optimal filtering, innovation process, conditionally Gaussian.

(Résumé : tsvp)

* IPPI, Russian Academy of Sciences, Moscow 101447, Russia, marina@sci.lpi.ac.ru, her stay in France was supported by INRIA, Marseille-Provence-Métropole and the Université Joseph Fourier.

** Idopt (CNRS, UJF, INPG, Inria) LMC-ImAG, BP 53, 38041 Grenoble Cedex 9, France, Alain.Le-Breton@imag.fr

*** CMI 39 rue Joliot-Curie, 13453 Marseille Cedex 13, France, mcroubau@sophia.inria.fr

Unité de recherche INRIA Sophia Antipolis 2004 route des Lucioles, BP 93, 06902 SOPHIA ANTIPOLIS Cedex (France)

Téléphone : 0493657777 - Télécopie : 0493657765 


\section{Une approche élémentaire pour le filtrage dans des systèmes avec bruit d'observation brownien fractionnaire}

Résumé : On considère le problème du filtrage optimal pour des systèmes linéaires et non linéaires avec bruit d'observation brownien fractionnaire. Une approche élémentaire et complètement autocontenue est développée. D'abord on démontre un résultat du type Girsanov et on introduit un processus qui joue ici le rôle que joue le processus d'innovation dans la situation usuelle où le bruit d'observation est un mouvement brownien. Ensuite l'approche

de Zakai est partiellement étendue pour obtenir certaines des équations du filtrage lorsque le processus d'état est une diffusion. Enfin, le cas spécifique des systèmes linéaires conditionnellement gaussiens est analysé. Un système d'équations fermé est obtenu pour la moyenne du filtre optimal et la variance conditionnelle de l'erreur de filtrage. Dans divers cas particuliers, les résultats sont complètement explicites.

Mots-clé : Mouvement brownien fractionnaire, formule de Girsanov, filtrage optimal, processus d'innovation, conditionnellement gaussien. 


\section{Contents}

1 Introduction $\quad 5$

2 Preliminaries $\quad 6$

3 A Girsanov type formula $\quad 12$

$\begin{array}{lll}4 & \text { The innovation type process } & 17\end{array}$

5 Optimal filtering of a diffusion process in fractional Brownian $\begin{array}{ll}\text { observation noise } & 24\end{array}$

6 Optimal filtering in conditionally Gaussian linear systems with fractional Brownian observation noise

$\mathrm{RR} \mathrm{n}^{\circ} 3439$ 
INRIA 


\section{Introduction}

Numerous theoretical results and successful applications have been already reported about stochastic models and modeling for long-range dependent phenomena (see e.g. Beran $[1,2]$ and references therein). The fractional Brownian motion (fBm for short), which provides a suitable generalization of the Brownian motion, is one of the simplest stochastic processes exhibiting long-range dependence. It has been widely used as a modeling tool (see e.g. Hurst [10, 11], Mandelbrot and Van Ness [23], Leland et al. [19], Norros [24, 25], Coelho and Decreusefond [4], Comte [3] and Dai and Heyde [6]). So in particular linear systems driven by fBm constitute a favorable ground for attempts to extend the classical theory of continuous-time stochastic systems driven by Brownian motions as developed for example in Liptser and Shiryaev [17]. Recently several contributions in this direction have been obtained for instance by Gripenberg and Norros [9] for a prediction problem, by Norros et al. [26] and Le Breton [15] for parameter estimation problems.

The filtering problem for systems driven by fBm's has also been studied. Various specific cases were investigated by Kleptsyna et al. [12, 13, 14] and Le Breton [15]. Recently it has been addressed in a rather general framework by Coutin and Decreusefond [5], but the special form of involved equations for the system dynamics allowed them to make use only of the classical Girsanov's formula for the Brownian motion. Here the filtering problem is revisited in a simple setting, yet not already covered, for which an appropriate Girsanov type formula for $\mathrm{fBm}$ is needed. A quite elementary and completely self-contained approach is developed to derive an explicit solution (see also Le Breton [16] for preliminary results). We consider real-valued continuous processes $X=$ $\left(X_{t}, t \in[0, T]\right)$ and $Y=\left(Y_{t}, t \in[0, T]\right)$, representing the signal and the observation respectively. The observation equation for the signal $X$ is the following :

$$
Y_{t}=\xi+\int_{0}^{t} A\left(s, X_{s}\right) d s+\int_{0}^{t} B(s) d W_{s}, t \in[0, T]
$$

Here $W=\left(W_{t}, t \in[0, T]\right)$ is a $\mathrm{fBm}$ with Hurst parameter $H \in\left(\frac{1}{2}, 1\right)$. The coefficients $A$ and $B$ are continuous functions of $[0, T] \times \mathbb{R}$ and $[0, T]$ respectively into $\mathbb{R}$ and $B$ is supposed nonvanishing. Moreover the random initial

$\mathrm{RR} \mathrm{n}^{\circ} 3439$ 
condition $\xi$ in $(1)$ is such that the pair $(X, \xi)$ is independent of $W$ and has a given distribution $\mu_{(X, \xi)}$.

Supposing that only $Y$ is observed but one wishes to know $X$, the classical problem of filtering the signal $X$ at time $t$ from the observation of $Y$ up to time $t$ occurs. The solution to this problem is the conditional distribution $\pi_{t}$ of $X_{t}$ given $\mathcal{Y}_{t}=\sigma\left(\left\{Y_{s}, 0 \leq s \leq t\right\}\right)$ which is called the optimal filter. The most successful result of this kind, the so-called Kalman-Bucy filter, was obtained for linear systems driven by Brownian motions in early sixties. It was followed by many contributions extending the result to such nonlinear systems. There are now several efficient strategies to deal with the filtering problem, extensively developed for $X$ and $Y$ semimartingales (see e.g. Liptser and Shiryaev [17] and Elliott [8]). The aim of the present work is to show that actually similar developments are possible in the case of fractional Brownian noises.

The paper is organized as follows. At first in Section 2 some notations are fixed and preliminaries about $\mathrm{fBm}$ and integration with respect to $\mathrm{fBm}$ are discussed. In particular integral transformations which change a $\mathrm{fBm}$ to martingales are described. Then in Section 3 an elementary approach is proposed to derive an appropriate Girsanov type formula. This approach, which extends that initiated in [26] and [15, 16], permits to avoid resorting to very elaborated tools as those developed in Decreusefond and Üstünel [7] and used in [5]. In Section 4 a complementary preparation for filtering deals with integral representations of various processes involved in the problem. At this end a process which plays here the role of the innovation process in the classical situation where the noises are Brownian motions is introduced. Then in Section 5 the Girsanov type formula and the representation results are used to derive filtering equations when the signal process is a diffusion. Conditionally Gaussian linear systems are analyzed in Section 6. Closed form equations are derived both for the mean of the optimal filter and the conditional variance of the filtering error.

\section{Preliminaries}

Here, for some fixed $T>0, W=\left(W_{t}, t \in[0, T]\right)$ is a normalized fractional Brownian motion on $[0, T]$ with Hurst parameter $H \in(1 / 2,1)$ means that $W$ 
is a process, defined on some probability space $(\Omega, \mathcal{F}, \mathbb{P})$, with the following properties:

(i) $W$ is a Gaussian process with continuous paths and stationary increments,

(ii) $W_{0}=0$ and $\mathbb{E} W_{t}=0, \mathbb{E} W_{t}^{2}=t^{2 H}$ for all $t \in[0, T]$.

Of course the $\mathrm{fBm}$ reduces to the standard Brownian motion when $H=\frac{1}{2}$. Actually the $\mathrm{fBm}$ can be obtained as a stochastic integral with respect to a standard Brownian motion (see e.g. Mandelbrot and Van Ness [23], where the fBm was given its name, Samorodnitsky and Taqqu [28], [26] and [7]). Nevertheless the $\mathrm{fBm}$ is not a semimartingale (see e.g. Lin [20]) and hence the theory of integration with respect to $\mathrm{fBm}$ does not fall into the classical stochastic integration theory. However the special case of a deterministic integrand turns out to be both easy to handle and sufficient for the purpose of the present paper. Both $L^{2}$ and pathwize approaches are possible (see e.g. [9] and [26] for an elementary calculation-oriented introduction).

Let us describe briefly the technical framework. Let $\Gamma_{H}$ denote the integral operator

$$
\Gamma_{H} f(t)=H(2 H-1) \int_{0}^{T} f(s)|s-t|^{2 H-2} d s, t \in[0, T]
$$

and define the inner product

$$
\begin{aligned}
<<f, g>>_{H} & =<<f, \Gamma_{H} g>> \\
& =H(2 H-1) \int_{0}^{T} \int_{0}^{T} f(s) g(t)|s-t|^{2 H-2} d s d t,
\end{aligned}
$$

where $<<.>>$ stands for the usual inner product on $L^{2}([0, T])$. Let $L_{H}^{2}$ be the space of equivalence classes of measurable functions $f$ on $[0, T]$ such that $<<f, f>>_{H}<\infty$. Now it is easy to check that the association $\mathbb{1}_{[0, t)} \rightarrow W_{t}$ can be extended to an isometry between the function space $L_{H}^{2}$ and the Gaussian space generated by the random variables $W_{t}, t \geq 0$, as the smallest closed linear subspace of $L^{2}(\Omega, \mathcal{F}, \mathbb{P})$ containing them. For $f \in L_{H}^{2}$, the integral $\int_{0}^{T} f(s) d W_{s}$ can now be defined as the image of $f$ in this isometry. Of course, for any $f$

$\mathrm{RR} \mathrm{n}^{\circ} 3439$ 
and $g$ in $L_{H}^{2}$, the following identity holds:

$$
\mathbb{E}\left[\left\{\int_{0}^{T} f(s) d W_{s}\right\}\left\{\int_{0}^{T} g(t) d W_{t}\right\}\right]=<<f, g>_{H},
$$

where $<<f, g>>_{H}$ is given by (2). Actually if the function $f$ is of bounded variation, then the integral $\int_{0}^{T} f(s) d W_{s}(\omega)$ can be defined for almost all $\omega$ 's as a limit of Riemann sums, and it is easy to see that the integral obtained coincides with the $L^{2}$ integral almost surely. The convergence of these Riemann sums is equivalent to that of the Riemann-Stieltjes type integral appearing in the right hand side of the integration by parts formula

$$
\int_{0}^{T} f(s) d W_{s}=f(T) W_{T}-\int_{0}^{T} W_{s} d f(s)
$$

and thus guaranteed by the continuity of the sample paths of $W$. Much stronger results (cf. e.g. Lyons [22]) can be obtained using more precise properties of the sample paths of the $\mathrm{fBm}$. Of course, for an appropriate function $f$, the stochastic process $\left(\int_{0}^{t} f(s) d W_{s}, t \in[0, T]\right)$, integral of $f$ with respect to $W$, is then defined by

$$
\int_{0}^{t} f(s) d W_{s} \triangleq \int_{0}^{T} \mathbb{1}_{[0, t)}(s) f(s) d W_{s}, \quad t \in[0, T],
$$

and a continuous version of this process can be chosen, what shall be always done in the sequel.

Although the $\mathrm{fBm}$ is not a semimartingale, simple integral transformations which change it to martingales can be identified. To prove a Girsanov type formula in the next section, we shall make use of the following technical result which was shown in [15] with slightly different notations (see also [26] for a particular case). In the sequel $\Gamma$ stands for the gamma function.

Lemma 1 Let $W$ be a fBm with Hurst parameter $H \in(1 / 2,1)$. Let $C$ be a continuous function of $[0, T]$ into $\mathbb{R}$. Define for $0<t \leq T$ the function $k_{C}^{t}=\left(k_{C}^{t}(s), 0<s<t\right)$ by

$k_{C}^{t}(s) \triangleq-\rho_{H}^{-1} s^{\frac{1}{2}-H} \frac{d}{d s} \int_{s}^{t} d w w^{2 H-1}(w-s)^{\frac{1}{2}-H} \frac{d}{d w} \int_{0}^{w} d z z^{\frac{1}{2}-H}(w-z)^{\frac{1}{2}-H} C(z)$, 
where $\rho_{H}=\Gamma^{2}\left(\frac{3}{2}-H\right) \Gamma(2 H+1) \sin (\pi H)$. Then the function $k_{C}^{t}$ satisfies the equation

$$
H(2 H-1) \int_{0}^{t} k_{C}^{t}(s)|s-r|^{2 H-2} d s=C(r) ; 0<r<t .
$$

Define for $0 \leq t \leq T$

$$
N_{t}^{C} \triangleq \int_{0}^{t} k_{C}^{t}(s) d W_{s} ; \quad<N^{C}>_{t} \triangleq \int_{0}^{t} C(s) k_{C}^{t}(s) d s .
$$

Then the process $N^{C}=\left(N_{t}^{C}, 0 \leq t \leq T\right)$ is a Gaussian martingale and the function $\left\langle N^{C}\right\rangle=\left(\left\langle N^{C}\right\rangle_{t}, t \in[0, T]\right)$ is its variance function.

Remark 1 (a) Let us mention that the definition (4) of the function $k_{C}^{t}$ can be interpreted in terms of right and left fractional derivatives $\mathcal{D}_{0^{+}}^{H-\frac{1}{2}}$ and $\mathcal{D}_{t^{-}}^{H-\frac{1}{2}}$ (see Samko et al. [27]) :

$$
k_{C}^{t}(s)=[\Gamma(2 H+1) \sin (\pi H)]^{-1} s^{\frac{1}{2}-H} \mathcal{D}_{t^{-}}^{H-\frac{1}{2}} s^{2 H-1} \mathcal{D}_{0^{+}}^{H-\frac{1}{2}} s^{\frac{1}{2}-H} C(s) ; 0<s<t .
$$

(b) In particular, for $C \equiv 1$ the function $k_{C}^{t}$ is (see [26] or [15]) :

$$
k_{*}^{t}(s)=\kappa_{H}^{-1} s^{\frac{1}{2}-H}(t-s)^{\frac{1}{2}-H}, 0<s<t,
$$

with

$$
\kappa_{H}=2 H \Gamma\left(\frac{3}{2}-H\right) \Gamma\left(H+\frac{1}{2}\right) .
$$

Then the corresponding Gaussian martingale (called in [26] the "fundamental martingale" associated to the $\mathrm{fBm} W$ ) is

$$
N_{t}^{*}=\kappa_{H}^{-1} \int_{0}^{t} s^{\frac{1}{2}-H}(t-s)^{\frac{1}{2}-H} d W_{s}, t \in[0, T],
$$

and it has the variance function

$$
<N^{*}>_{t}=\kappa_{H}^{-1} \int_{0}^{t} s^{\frac{1}{2}-H}(t-s)^{\frac{1}{2}-H} d s=\lambda_{H}^{-1} t^{2-2 H}, t \in[0, T],
$$

where

$$
\lambda_{H}=\frac{2 H \Gamma(3-2 H) \Gamma\left(H+\frac{1}{2}\right)}{\Gamma\left(\frac{3}{2}-H\right)} .
$$

$\mathrm{RR} \mathrm{n}^{\circ} 3439$ 
The following property, which is proved in [26], will be a key point in our developments below : the filtration generated by the martingale $N^{*}$ coincides with that generated by the $\mathrm{fBm} W$ up to sets of $\mathbb{P}$ probability zero.

(c) If $C$ and $D$ are two continuous functions of $[0, T]$ into $\mathbb{R}$ and processes $N^{C}$ and $N^{D}$ correspond to $C$ and $D$ respectively by (6), then of course the pair $\left(N^{C}, N^{D}\right)$ is Gaussian; actually, from (3) and (5), one may easily check that it is a martingale with the covariance function

$$
<N^{C}, N^{D}>_{t}=\int_{0}^{t} k_{C}^{t}(s) D(s) d s=\int_{0}^{t} k_{D}^{t}(s) C(s) d s, t \in[0, T] .
$$

In particular

$$
<N^{C}, N^{*}>_{t}=\int_{0}^{t} k_{C}^{t}(s) d s=\int_{0}^{t} k_{*}^{t}(s) C(s) d s, t \in[0, T] .
$$

(d) Let us observe that when $H=\frac{1}{2}$, i.e. $W$ is a standard Brownian motion, then assertions in Lemma 1 reduce to elementary facts concerning the Itô stochastic integral with respect to $W$. Actually in this case

$$
k_{C}^{t} \equiv C ; N_{t}^{C}=\int_{0}^{t} C(s) d W_{s} ;<N^{C}>_{t}=\int_{0}^{t} C^{2}(s) d s,
$$

and in particular $k_{*}^{t} \equiv 1, N_{t}^{*}=W_{t},<N^{*}>_{t}=t$.

Now we state some useful complementary properties of martingales defined as in Lemma 1.

Lemma 2 Let assumptions and notations be the same as in Lemma 1. Let also $N^{*}$ be the martingale defined by (8) and $\left\langle N^{*}\right\rangle$ be its variance function defined by (9). Then the martingale $N^{C}$ defined by (6) is continuous. Moreover there exists a measurable function $q^{C}=\left(q_{t}^{C}, t \in[0, T]\right)$ such that

$$
\int_{0}^{T}\left(q_{t}^{C}\right)^{2} d<N^{*}>_{t}<+\infty
$$

and the representations

$$
N_{t}^{C}=\int_{0}^{t} q_{s}^{C} d N_{s}^{*} ;<N^{C}>_{t}=\int_{0}^{t}\left(q_{s}^{C}\right)^{2} d<N^{*}>_{s}, t \in[0, T]
$$

hold. 
Proof At first let us discuss the continuity property of $N^{C}$. Since from Lemma $1 N^{C}$ is a Gaussian martingale, it is sufficient to show that its variance function $\left\langle N^{C}\right\rangle$ is continuous. Actually this can be easily proved starting from the definition (6) of this function and the formula (4) for the involved function $k_{C}^{t}$.

Of course in particular $N^{*}$ is continuous. Moreover, from Remark 1(b), $N^{*}$ generates the same filtration as the $\mathrm{fBm} W$ up to sets of $\mathbb{P}$ probability zero. Therefore, since $N^{C}$ is clearly adapted to the last filtration, it is also adapted to the filtration generated by $N^{*}$. Consequently, since from Remark 1 (c) the pair $\left(N^{C}, N^{*}\right)$ is a Gaussian martingale, we may apply the generalized Kalman filter derived in Liptser and Shiryaev [18] (see Problem 2, p. 320) and hence get that the representations (11) hold with

$$
q_{t}^{C}=\frac{d<N^{C}, N^{*}>_{t}}{d<N^{*}>_{t}}, t \in[0, T]
$$

where $\left\langle N^{C}, N^{*}>\right.$ is the covariance function of $\left(N^{C}, N^{*}\right)$ given by (10).

Remark 2 (a) Let us observe that, taking into account (9), an immediate consequence of equation (12) is that the function $\left\langle N^{C}, N^{*}\right\rangle$ given by (10) is absolutely continuous with respect to the Lebesgue measure with the derivative $t \rightarrow \lambda_{H}^{-1}(2-2 H) t^{1-2 H} q_{t}^{C}$. Moreover, due to the expression (7) of the function $k_{*}^{t}$, this derivative can be seen also as the right fractional derivative $\mathcal{D}_{0^{+}}^{H-\frac{1}{2}}$ (see [27]) of the function $t \rightarrow\left[2 H \Gamma\left(H+\frac{1}{2}\right)\right]^{-1} t^{\frac{1}{2}-H} C(t)$. Finally we can write

$$
\int_{0}^{t} k_{*}^{t}(s) C(s) d s=\int_{0}^{t} q_{s}^{C} d<N^{*}>_{s}, t \in[0, T]
$$

where

$$
q_{t}^{C}=\frac{\Gamma(2-2 H)}{\Gamma\left(\frac{3}{2}-H\right)} t^{2 H-1} \mathcal{D}_{0^{+}}^{H-\frac{1}{2}} t^{\frac{1}{2}-H} C(t), t \in[0, T] .
$$

(b) More generally, for any fixed $u \in(0, T)$, we can write

$$
\int_{u}^{t} k_{*}^{t}(s) C(s) d s=\int_{u}^{t} q^{C}(s, u) d<N^{*}>_{s}, t \in[u, T]
$$

where

$$
q^{C}(t, u)=\frac{\Gamma(2-2 H)}{\Gamma\left(\frac{3}{2}-H\right)} t^{2 H-1} \mathcal{D}_{u^{+}}^{H-\frac{1}{2}} t^{\frac{1}{2}-H} C(t), t \in[u, T]
$$

$\mathrm{RR} \mathrm{n}^{\circ} 3439$ 
Hence in the sequel we shall use the notation $q^{C}(t, 0)$ for $q_{t}^{C}$.

(c) Let us also mention that when $H=\frac{1}{2}$, i.e. $W$ is a standard Brownian motion, then of course the assertion in Lemma 2 reduces to a triviality with $q^{C} \equiv C$. Actually in this case, with the notation of Remark (b) just above, we have more generally $q^{C}(., u) \equiv C$.

Now we turn to some complementary preparation for studying the filtering problem.

\section{A Girsanov type formula}

There is now a quite usual strategy to deal with the filtering problem, extensively developed for $X$ and $Y$ semimartingales, i.e. when in particular the observation noise $W$ in (1) is a Brownian motion which means $H=\frac{1}{2}$. The first step consists in constructing a new probability measure $\widetilde{\mathbb{P}}$, called the reference probability, a $\widetilde{\mathbb{P}}$ Brownian motion carrying the same filtration as the observation process and such that under $\widetilde{\mathbb{P}}$, it is independent of the signal process. The second step is to show that the optional projection under $\mathbb{P}$ can be transformed to a $\widetilde{\mathbb{P}}$ conditional expectation to obtain an equation for

$$
\pi_{t}(\phi) \triangleq \mathbb{E}\left(\phi\left(X_{t}\right) \mid \mathcal{Y}_{t}\right)
$$

for sufficiently regular function $\phi$ from $\mathbb{R}$ to $\mathbb{R}$. We shall show that in the situation which we are dealing with here a similar approach can be led. A key point is the construction of the appropriate reference probability which relies on a Girsanov type result. In [7], Girsanov's formula was derived in a very general framework for changes of measure in a stochastic basis with $\mathrm{fBm}$ by making use of rather sophisticated tools. Here, due to our simple setting, we may develop an elementary approach which leads to quite explicit results (see [26] and $[15,16]$ for more specific cases).

Actually in the sequel it is convenient to work on the canonical space of the process $(X, Y)$. Let $\Omega=\mathcal{C}\left([0, T] ; \mathbb{R}^{2}\right)$ be the space of continuous functions from $[0, T]$ into $\mathbb{R}^{2}$. Consider also the canonical process $\left(X, W^{*}\right)=$ $\left(\left(X_{t}, W_{t}^{*}\right), t \in[0, T]\right)$ on $\Omega$ where $\left(X_{t}, W_{t}^{*}\right)(x, y)=\left(x_{t}, y_{t}\right)$ for any $(x, y) \in \Omega$. 
Hereafter $\widetilde{\mathbb{P}}$ denotes the unique probability measure on $\Omega$ such that defining the variable $\xi$ by

$$
\xi=W_{0}^{*},
$$

and the process $\left.\widetilde{W}=\left(\widetilde{W}_{t}\right), t \in[0, T]\right)$ by

$$
\widetilde{W}_{t}=W_{t}^{*}-W_{0}^{*}, t \in[0, T]
$$

the following properties hold :

- the pair $(X, \xi)$ is independent of $\widetilde{W}$ and has the distribution $\mu_{(X, \xi)}$,

- the process $\widetilde{W}$ is a fBm with Hurst parameter $H$.

The canonical filtration on $\Omega$ is $\left(\mathcal{F}_{t}, t \in[0, T]\right)$ where $\mathcal{F}_{t}=\sigma\left(\left\{\left(X_{s}, W_{s}^{*}\right), 0 \leq\right.\right.$ $s \leq t\}) \vee \mathcal{N}, \mathcal{N}$ denoting the set of null sets of $(\Omega, \widetilde{\mathbb{P}})$.

Let us define for all continuous function $x=\left(x_{t}, t \in[0, T]\right)$ the function $C(x)$ on $[0, T]$ by

$$
C(x)(t) \triangleq \frac{A\left(t, x_{t}\right)}{B(t)}, t \in[0, T] .
$$

Consider, for any $t \in[0, T]$, the function $k_{C(x)}^{t}=\left(k_{C(x)}^{t}(s), 0<s<t\right)$ defined from equation (4) with $C(x)$ in place of $C$. Define also the process $\widetilde{N}(x)=$ $\left(\widetilde{N}_{t}(x), t \in[0, T]\right)$ by

$$
\widetilde{N}_{t}(x) \triangleq \int_{0}^{t} k_{C(x)}^{t}(s) d \widetilde{W}_{s}, t \in[0, T]
$$

and the function $<\widetilde{N}>(x)=\left(<\widetilde{N}>_{t}(x), t \in[0, T]\right)$ by

$$
<\widetilde{N}>_{t}(x) \triangleq \int_{0}^{t} k_{C(x)}^{t}(s) C(x)(s) d s, t \in[0, T]
$$

which actually is nothing but $\left\langle N^{C(x)}>\right.$ defined from the second part of (6) with $C(x)$ in place of $C$.

Observe that from Lemma 1 , under $\widetilde{\mathbb{P}}$ the process $\widetilde{N}(x)$ is a Gaussian martingale with the variance function $\langle\widetilde{N}>(x)$. Then of course defining for all $t \in[0, T]$

$$
\Lambda_{t}(x) \triangleq \exp \left\{\widetilde{N}_{t}(x)-\frac{1}{2}<\widetilde{N}>_{t}(x)\right\}
$$

$\mathrm{RR} \mathrm{n}^{\circ} 3439$ 
the following equality holds :

$$
\widetilde{\mathbb{E}}\left(\Lambda_{t}(x)\right)=1
$$

Finally, for any $t \in[0, T]$, we introduce the random variables

$$
\widetilde{N}_{t} \triangleq \widetilde{N}_{t}(X) ; \quad<\widetilde{N}>_{t} \triangleq<\widetilde{N}>_{t}(X)
$$

and

$$
\Lambda_{t} \triangleq \Lambda_{t}(X)=\exp \left\{\widetilde{N}_{t}-\frac{1}{2}<\widetilde{N}>_{t}\right\}
$$

Let us emphasize that actually for each $t$ the variables $N_{t},\langle N\rangle_{t}$ and $\Lambda_{t}$ depend only on values of $X$ in the past of time $t$ i.e. for instance $N_{t}=N_{t}\left(X^{(t)}\right)$ where $X^{(t)}=\left(X_{s}, 0 \leq s \leq t\right)$. Now, since under $\widetilde{\mathbb{P}}$ the processes $X$ and $\widetilde{W}$ are independent, making use of identity (19), it is readily seen that $\widetilde{\mathbb{E}}\left(\Lambda_{t}\right)=1$ and $\widetilde{\mathbb{E}}\left(\Lambda_{T} \mid \mathcal{F}_{t}\right)=\Lambda_{t}$ for all $t \in[0, T]$. Hence we may define the probability $\mathbb{P}$ which is absolutely continuous with respect to $\widetilde{\mathbb{P}}$ with the Radon-Nikodym derivative $\Lambda_{T}$, so that for any $t \in[0, T]$ the local density process on the family $\left\{\mathcal{F}_{t}, t \in[0, T]\right\}$ is nothing but $\left(\Lambda_{t}, t \in[0, T]\right)$.

Now, defining on $\left(\Omega,\left(\mathcal{F}_{t}\right), \widetilde{\mathbb{P}}\right)$ the process $W=\left(W_{t}, t \in[0, T]\right)$ by

$$
W_{t} \triangleq \widetilde{W}_{t}-\int_{0}^{t} C(X)(s) d s, t \in[0, T]
$$

and considering the continuous adapted process $Y=\left(Y_{t}, t \in[0, T]\right)$ given by :

$$
Y_{t}=\xi+\int_{0}^{t} B(s) d \widetilde{W}_{s}, t \in[0, T]
$$

we may state the following Girsanov type result :

Proposition 1 Let $\mathbb{P}$ be the probability measure $\mathbb{P} \triangleq \Lambda_{T} \cdot \widetilde{\mathbb{P}}$ where $\Lambda_{T}$ is given by (21). Then, under $\mathbb{P}$, the distribution of the system $(X, \xi, W)$, where $W$ is defined by (22), is the same as that of the system $(X, \xi, \widetilde{W})$ under $\widetilde{\mathbb{P}}$. Moreover under $\mathbb{P}$ the process $Y$ defined by (23) satisfies the equation (1). 
Proof To prove the first assertion it is sufficient to show that for any fixed $0 \leq t_{1}<t_{2} \ldots<t_{n} \leq T$, the distribution of $\left(X, \xi, W_{t_{1}}, \ldots, W_{t_{n}}\right)$ with respect to $\mathbb{P}$ is that of $\left(X, \xi, \widetilde{W}_{t_{1}}, \ldots, \widetilde{W}_{t_{n}}\right)$ with respect to $\widetilde{\mathbb{P}}$. So we fix Borel sets $\Gamma$ and $\Delta$ in $C([0, T] ; \mathbb{R})$ and $\mathbb{R}$ respectively and a vector $\beta=\left(\beta_{1}, \ldots, \beta_{n}\right)^{\prime}$ in $\mathbb{R}^{n}$. We have to check that

$$
\mathbb{E}\left(\mathbb{1}_{\Gamma \times \Delta}(X, \xi) \exp \left(\sum_{1}^{n} \beta_{i} W_{t_{i}}\right)\right)=\mu_{(X, \xi)}(\Gamma \times \Delta) \exp \left(\frac{1}{2}\left[\beta^{\prime} \Sigma_{H}^{(n)} \beta\right]\right),
$$

where $\Sigma_{H}^{(n)}$ stands for the covariance matrix of $\left.\left(\widetilde{W}_{t_{1}}, \ldots, \widetilde{W}_{t_{n}}\right)^{\prime}\right)$ with respect to $\widetilde{\mathbb{P}}$. By definitions $(22)$ and $(21)$ of $W$ and $\Lambda_{T}$ respectively, from $\mathbb{P}=\Lambda_{T} \cdot \widetilde{\mathbb{P}}$ we compute the left hand side of (24) $(\operatorname{lhs}(24)$ for short) as

$$
\widetilde{\mathbb{E}}\left(\mathbb{1}_{\Gamma \times \Delta}(X, \xi) \exp \left\{\sum_{1}^{n} \beta_{i} \widetilde{W}_{t_{i}}-\sum_{1}^{n} \beta_{i} \int_{0}^{t_{i}} C(X)(u) d u+\widetilde{N}_{T}-\frac{1}{2}<\widetilde{N}>_{T}\right\}\right) .
$$

Then, taking into account the independence of $(X, \xi)$ and $\widetilde{W}$ with respect to $\widetilde{\mathbb{P}}$, it comes that

$$
\operatorname{lhs}(24)=\widetilde{\mathbb{E}}\left(\mathbb{1}_{\Gamma \times \Delta}(X, \xi)[\widetilde{\mathbb{E}}(\exp (U(x)))]_{\mid x=X}\right),
$$

where, for a continuous function $x$ of $[0, T]$ into $\mathbb{R}, U(x)$ is the random variable

$$
U(x)=\sum_{1}^{n} \beta_{i} \widetilde{W}_{t_{i}}-\sum_{1}^{n} \beta_{i} \int_{0}^{t_{i}} C(x)(u) d u+\widetilde{N}_{T}(x)-\frac{1}{2}<\widetilde{N}>_{T}(x),
$$

with $\widetilde{N}_{T}(x)$ and $<\widetilde{N}>_{T}(x)$ given by (16) and (17) respectively. With respect to $\widetilde{\mathbb{P}}$ the random variable $U(x)$ is Gaussian with mean

$$
\widetilde{\mathbb{E}}(U(x))=-\sum_{1}^{n} \beta_{i} \int_{0}^{t_{i}} C(x)(u) d u-\frac{1}{2}<\widetilde{N}>_{T}(x)
$$

and variance

$$
\widetilde{\operatorname{var}}(U(x))=\widetilde{\mathbb{E}}\left[\left(\widetilde{N}_{T}(x)\right)^{2}\right]+\widetilde{\mathbb{E}}\left[\left(\sum_{1}^{n} \beta_{i} W_{t_{i}}\right)^{2}\right]+2 \sum_{1}^{n} \beta_{i} \widetilde{\mathbb{E}}\left(\widetilde{N}_{T}(x) \widetilde{W}_{t_{i}}\right),
$$

or

$$
\widetilde{\operatorname{var}}(U(x))=<\widetilde{N}>_{T}(x)+\beta^{\prime} \Sigma_{H}^{(n)} \beta+2 \sum_{1}^{n} \beta_{i} \widetilde{\mathbb{E}}\left(\widetilde{N}_{T}(x) \widetilde{W}_{t_{i}}\right)
$$

$\mathrm{RR} \mathrm{n}^{\circ} 3439$ 
But, from identity (3), definition (16) of $\widetilde{N}_{T}(x)$ and equation (5), it is easy to check that

$$
\widetilde{\mathbb{E}}\left(\widetilde{N}_{T}(x) \widetilde{W}_{t_{i}}\right)=\int_{0}^{t_{i}} C(x)(u) d u
$$

Finally we get

$$
\widetilde{\operatorname{var}}(U(x))=<\widetilde{N}>_{T}(x)+\beta^{\prime} \Sigma^{(n)} \beta+2 \sum_{1}^{n} \beta_{i} \int_{0}^{t_{i}} C(x)(u) d u .
$$

But, since under $\widetilde{\mathbb{P}}$ the variable $U(x)$ is Gaussian, we have

$$
\widetilde{\mathbb{E}}[\exp (U(x))]=\exp \left[\widetilde{\mathbb{E}}\left(U(x)+\frac{1}{2} \widetilde{\operatorname{var}}(U(x))\right]\right.
$$

Hence, from equations (26) and (27), it is readily seen that

$$
\widetilde{\mathbb{E}}[\exp (U(x))]=\exp \left(\frac{1}{2} \beta^{\prime} \Sigma_{H}^{(n)} \beta\right)
$$

and inserting this in (25) we obtain that

$$
\operatorname{lhs}(24)=\left[\widetilde{\mathbb{E}}\left(\mathbb{1}_{\Gamma \times \Delta}(X, \xi)\right)\right] \exp \left(\frac{1}{2} \beta^{\prime} \Sigma_{H}^{(n)} \beta\right),
$$

which means that the equality (24) holds.

Finally the second assertion in the statement is a straightforward consequence of the first one and of definition (22) of the process $W$.

We remind now the usual notations which we use hereafter :

- $\mathcal{Y}_{t}=\sigma\left(\left\{Y_{s}, 0 \leq s \leq t\right\}\right), \quad t \in[0, T]$,

- $\pi_{t}(\phi)=\mathbb{E}\left[\phi\left(X_{t}\right) \mid \mathcal{Y}_{t}\right] ; \quad \sigma_{t}(\phi)=\tilde{\mathbb{E}}\left[\phi\left(X_{t}\right) \Lambda_{t} \mid \mathcal{Y}_{t}\right], \quad t \in[0, T]$

The distribution $\pi_{t}$ is the optimal filter (when observation is made on $[0, t]$ ) whereas the measure $\sigma_{t}$ is the so-called unnormalized filter. They both operate on bounded continuous real-valued functions and the well-known Bayes type formula, called the Kallianpur-Striebel formula, holds : for all $t \in[0, T], \widetilde{\mathbb{P}}$ and $\mathbb{P}$ almost everywhere,

$$
\pi_{t}(\phi)=\frac{\sigma_{t}(\phi)}{\sigma_{t}(1)}
$$


Actually, more generally, for a sufficiently regular function $\psi_{t}$ on the $\operatorname{set} \mathcal{C}([0, t] ; \mathbb{R})$ of continuous functions on $[0, t]$, we shall also be concerned with similar quantities with the variable $\psi_{t}\left(X^{(t)}\right)$, written simply $\psi_{t}(X)$, in place of $\phi\left(X_{t}\right)$, where $X^{(t)}$ stands for the process $X^{(t)}=\left(X_{s}, 0 \leq s \leq t\right)$. So for instance the notation $\pi_{t}(\psi)$ will also be used for the conditional expectation $\mathbb{E}\left[\psi_{t}(X) \mid \mathcal{Y}_{t}\right]$.

\section{The innovation type process}

Here we deal with integral representations of various processes involved in the filtering problem. We start with the processes $N=\left(N_{t}, t \in[0, T]\right)$ and $<N>=\left(<N>_{t}, t \in[0, T]\right)$ defined from (6) by plugging in the process $C(X)$ in place of $C$ i.e.

$$
N_{t} \triangleq N_{t}^{C(X)} ; \quad<N>_{t} \triangleq<N^{C(X)}>_{t}, t \in[0, T] .
$$

Notice that here again actually $N_{t}$ and $\langle N\rangle_{t}$ depend only on values of $X^{(t)}=\left(X_{s}, 0 \leq s \leq t\right)$

Recall the martingale $N^{*}$ and the function $\left\langle N^{*}>\right.$ defined in Remark 1 (b) by (8) and (9) respectively i.e.

$$
N_{t}^{*} \triangleq \int_{0}^{t} k_{*}^{t}(s) d W_{s} ;<N^{*}>_{t} \triangleq \int_{0}^{t} k_{*}^{t}(s) d s, t \in[0, T],
$$

where the function $k_{*}^{t}$ is given by (7).

Now let us define the $\left(\mathcal{F}_{t}\right)$-adapted processes $\left\langle N, N^{*}\right\rangle=\left(<N, N^{*}\right\rangle_{t}, t \in$ $[0, T])$ and $q(X)=\left(q_{t}(X), t \in[0, T]\right)$ from (10) and (12) respectively by

$$
<N, N^{*}>_{t} \triangleq<N^{C(X)}, N^{*}>_{t}=\int_{0}^{t} k_{*}^{t}(s) C(X)(s) d s, t \in[0, T]
$$

and

$$
q_{t}(X) \triangleq q_{t}^{C(X)}=\frac{d<N, N^{*}>_{t}}{d<N^{*}>_{t}}, t \in[0, T] .
$$

Applying Lemma 2, substituting $C(X)$ to $C$, it is readily seen that $\mathbb{P}$ almost surely

$$
\int_{0}^{T} q_{t}^{2}(X) d<N^{*}>_{t}<+\infty
$$

$\mathrm{RR} \mathrm{n}^{\circ} 3439$ 
and the representations

$$
N_{t}=\int_{0}^{t} q_{s}(X) d N_{s}^{*} ;<N>_{t}=\int_{0}^{t} q_{s}^{2}(X) d<N^{*}>_{s}, t \in[0, T]
$$

hold.

Now we introduce the process $Z$ defined by

$$
Z_{t} \triangleq \int_{0}^{t} k_{C(X)}^{t}(s) B^{-1}(s) d Y_{s}, t \in[0, T]
$$

where $Y$ is the observation process.

Finally we define the process $Z^{*}$ by

$$
Z_{t}^{*} \triangleq \int_{0}^{t} k_{*}^{t}(s) B^{-1}(s) d Y_{s}, t \in[0, T]
$$

where the function $k_{*}^{t}$ is given by $(7)$.

Under $\mathbb{P}$, since from $(1) d Y_{t}=A\left(t, X_{t}\right) d t+B(t) d W_{t}$, the process $Z^{*}$ appears as a semimartingale with the following decomposition :

$$
Z_{t}^{*}=<N, N^{*}>_{t}+N_{t}^{*}, t \in[0, T],
$$

where $N^{*}$ is given by (30) and $\left\langle N, N^{*}\right\rangle$ is defined from (31).

Similarly the process $Z$ appears as a semimartingale with the decomposition :

$$
Z_{t}=<N>_{t}+N_{t}, t \in[0, T],
$$

where $N$ and $\langle N\rangle$ are given by (29). Now from (33), (32) and (36)-(37) we can write

$$
Z_{t}^{*}=\int_{0}^{t} q_{s}(X) d<N^{*}>_{s}+N_{t}^{*}, t \in[0, T]
$$

and

$$
Z_{t}=\int_{0}^{t} q_{s}^{2}(X) d<N^{*}>_{s}+\int_{0}^{t} q_{s}(X) d N_{s}^{*}, t \in[0, T] .
$$

Hence finally we get the following integral representation of $Z$ in terms of $Z^{*}$ :

$$
Z_{t}=\int_{0}^{t} q_{s}(X) d Z_{s}^{*}, t \in[0, T] .
$$


Now let us introduce the process $\nu=\left(\nu_{t}, t \in[0, T]\right)$ defined by

$$
\nu_{t}=Z_{t}^{*}-\int_{0}^{t} \pi_{s}(q) d<N^{*}>_{s}, t \in[0, T],
$$

as a candidate to play the role which plays the innovation process in the usual situation where the noises are Brownian motions. Recall the notation $\pi_{s}(q)=$ $\mathbb{E}\left(q_{s}(X) \mid \mathcal{Y}_{s}\right)$. We prove the following

Lemma 3 Let processes $Z^{*}$ and $\nu$ be defined by (35) and (40) respectively. Let the function $\left\langle N^{*}>\right.$ be given by (30) or (9). For $t \in[0, T]$, let $\mathcal{F}_{t}^{\xi, Z^{*}}$ denote the $\sigma$-field $\mathcal{F}_{t}^{\xi, Z^{*}}=\sigma\left(\left\{\xi ; Z_{s}^{*}, 0 \leq s \leq t\right\}\right)$. Then the following assertions hold:

(i) for all $t \in[0, T]$, the $\sigma$-fields $\mathcal{F}_{t}^{\xi, Z^{*}}$ and $\mathcal{Y}_{t}$ coincide up to sets of $\mathbb{P}$ probability zero,

(ii) under $\mathbb{P}$, the process $\nu$ is a continuous Gaussian $\left(\mathcal{Y}_{t}\right)$-martingale with the variance function $\left\langle N^{*}\right\rangle$,

(iii) if $M=\left(M_{t}, t \in[0, T]\right)$ is a square integrable $\left(\mathcal{Y}_{t}\right)$-martingale with respect to $\mathbb{P}, M_{0}=0$, then there exists a $\left(\mathcal{Y}_{t}\right)$-adapted process $\Gamma=\left(\Gamma_{t}, t \in\right.$ $[0, T])$ such that

$$
\mathbb{E}\left[\int_{0}^{T} \Gamma_{t}^{2} d<N^{*}>_{t}\right]<+\infty
$$

and $\mathbb{P}$ almost surely

$$
M_{t}=\int_{0}^{t} \Gamma_{s} d \nu_{s}, t \in[0, T]
$$

Proof At first let us prove assertion (i). Observe that under $\widetilde{\mathbb{P}}$, since $d Y_{s}=$ $B(s) d \widetilde{W}_{s}$ and $B$ is nonvanishing, we have $\mathcal{Y}_{t}=\sigma\left(\left\{\xi ; \widetilde{W}_{s}, 0 \leq s \leq t\right\}\right)$ up to sets of $\widetilde{\mathbb{P}}$ probability zero. But, under $\widetilde{\mathbb{P}}$, we have also almost surely

$$
Z_{t}^{*}=\int_{0}^{t} k_{*}^{t}(s) d \widetilde{W}_{s}, t \in[0, T]
$$

Then, from Remark $1(\mathrm{~b}), Z^{*}$ generates the same filtration as $\widetilde{W}$ up to sets of $\widetilde{\mathbb{P}}$ probability zero. Hence $\mathcal{Y}_{t}=\mathcal{F}_{t}^{\xi, Z^{*}}$ up to sets of $\widetilde{\mathbb{P}}$ probability zero.

$\mathrm{RR} \mathrm{n}^{\circ} 3439$ 
Consequently, since $\mathbb{P}$ is equivalent to $\widetilde{\mathbb{P}}$, assertion $(i)$ holds.

Now we turn to assertion (ii). From (40) and (38) we can write

$$
\nu_{t}=\int_{0}^{t}\left[q_{s}(X)-\pi_{s}(q)\right] d<N^{*}>_{s}+N_{t}^{*}, t \in[0, T] .
$$

Therefore, for $0 \leq s \leq t \leq T$ we have

$$
\nu_{t}-\nu_{s}=\int_{s}^{t}\left[q_{u}(X)-\pi_{u}(q)\right] d<N^{*}>_{u}+\left[N_{t}^{*}-N_{s}^{*}\right] .
$$

Then, exploiting the fact that $\left\langle N^{*}\right\rangle$ is deterministic, the conditional expectation $\mathbb{E}\left[\nu_{t}-\nu_{s} \mid \mathcal{Y}_{s}\right]$ can be computed as

$$
\mathbb{E}\left[\left(\int_{s}^{t} \mathbb{E}\left[q_{u}(X)-\pi_{u}(q) \mid \mathcal{Y}_{u}\right] d<N^{*}>_{u}\right) \mid \mathcal{Y}_{s}\right]+\mathbb{E}\left[\mathbb{E}\left(N_{t}^{*}-N_{s}^{*} \mid \mathcal{F}_{s}\right) \mid \mathcal{Y}_{s}\right]
$$

Now using the $\left(\left(\mathcal{F}_{t}\right), \mathbb{P}\right)$-martingale property of $N^{*}$ we get that

$$
\mathbb{E}\left[\nu_{t}-\nu_{s} \mid \mathcal{Y}_{s}\right]=0, \mathbb{P} \text { almost surely, }
$$

which gives that under $\mathbb{P}$, the process $\nu$ is a $\left(\mathcal{Y}_{t}\right)$-martingale. Moreover, with respect to the filtration $\left(\mathcal{F}_{t}\right), \nu$ is a continuous semimartingale. Hence, by the differentiation rule, we have the decomposition

$$
\nu_{t}^{2}=2 \int_{0}^{t} \nu_{s} d \nu_{s}+<N^{*}>_{t}, t \in[0, T] .
$$

This shows that, with respect to the filtration $\left(\mathcal{F}_{t}\right)$, the predictable quadratic variation of $\nu$ is nothing but the continuous deterministic function $\left\langle N^{*}\right\rangle$. Consequently, with respect to the filtration $\left(\mathcal{Y}_{t}\right)$, the predictable quadratic variation of $\nu$ is also $\left\langle N^{*}\right\rangle$ and thus the continuous $\left(\left(\mathcal{Y}_{t}\right), \mathbb{P}\right)$-martingale $\nu$ is necessarily Gaussian with the variance function $\left\langle N^{*}\right\rangle$.

Finally let us prove assertion (iii) in the lemma. From (40) we have

$$
Z_{t}^{*}=\int_{0}^{t} \pi_{s}(q) d<N^{*}>_{s}+\nu_{t}, t \in[0, T],
$$

where $\pi_{s}(q)$ is $\left(\mathcal{Y}_{t}\right)$-adapted and, from (ii), $\nu$ is a continuous Gaussian $\left(\left(\mathcal{Y}_{t}\right), \mathbb{P}\right)$ martingale such that $\langle\nu\rangle=\left\langle N^{*}\right\rangle$. Now let us define the $\left(\left(\mathcal{Y}_{t}\right), \mathbb{P}\right)$-martingale $\Lambda^{*}=\left(\Lambda_{t}^{*}, t \in[0, T]\right)$ by

$$
\Lambda_{t}^{*}=\exp \left\{-\int_{0}^{t} \pi_{s}(q) d \nu_{s}-\frac{1}{2} \int_{0}^{t} \pi_{s}^{2}(q) d<N^{*}>_{s}\right\}, t \in[0, T]
$$


and the probability measure $\mathbb{P}^{*}$ on $\Omega$ as $\mathbb{P}^{*}=\Lambda_{T}^{*} . \mathbb{P}$. Then, from the standard Girsanov theorem, the process $Z^{*}$ is a continuous Gaussian $\left(\left(\mathcal{Y}_{t}\right), \mathbb{P}^{*}\right)$-martingale such that $\left.<Z^{*}\right\rangle=<N^{*}>$.

Hence if $M^{*}=\left(M_{t}^{*}, t \in[0, T]\right)$ is a $\left(\left(\mathcal{Y}_{t}\right), \mathbb{P}^{*}\right)$-martingale, $M_{0}^{*}=0$, there exists a $\left(\mathcal{Y}_{t}\right)$-adapted process $g=\left(g_{t}, t \in[0, T]\right)$ such that $\mathbb{P}^{*}$ almost surely

$$
M_{t}^{*}=\int_{0}^{t} g_{s} d Z_{s}^{*}, t \in[0, T]
$$

Now if $M$ is a $\left(\left(\mathcal{Y}_{t}\right), \mathbb{P}\right)$-martingale, $M_{0}=0$, the process $M^{*}$ defined by

$$
M_{t}^{*}=\Lambda_{t}^{*-1} M_{t}, t \in[0, T],
$$

is a $\left(\left(\mathcal{Y}_{t}\right), \mathbb{P}^{*}\right)$-martingale, $M_{0}^{*}=0$. Hence it admits a representation of type (42), so that $\mathbb{P}$ almost surely

$$
M_{t}^{*}=\int_{0}^{t} g_{s} \pi_{s}(q) d<N^{*}>_{s}+\int_{0}^{t} g_{s} d \nu_{s}, t \in[0, T] .
$$

Since from (41) we have

$$
\Lambda_{t}^{*}=1-\int_{0}^{t} \Lambda_{s}^{*} \pi_{s}(q) d \nu_{s}, t \in[0, T]
$$

applying the Itô formula, for $t \in[0, T]$, we compute $M_{t}$ as $M_{t}=\Lambda_{t}^{*} M_{t}^{*}$ :

$$
\begin{aligned}
M_{t}= & \int_{0}^{t} M_{s}^{*} d \Lambda_{s}^{*}+\int_{0}^{t} \Lambda_{s}^{*} d M_{s}^{*}+<\Lambda^{*}, M^{*}>_{t} \\
= & -\int_{0}^{t} M_{s}^{*} \Lambda_{s}^{*} \pi_{s}(q) d \nu_{s}+\int_{0}^{t} \Lambda_{s}^{*} g_{s} \pi_{s}(q) d<N^{*}>_{s} \\
& \quad+\int_{0}^{t} \Lambda_{s}^{*} g_{s} d \nu_{s}-\int_{0}^{t} \Lambda_{s}^{*} g_{s} \pi_{s}(q) d<N^{*}>_{s} \\
= & \int_{0}^{t} \Lambda_{s}^{*}\left[g_{s}-M_{s}^{*} \pi_{s}(q)\right] d \nu_{s} .
\end{aligned}
$$

This means that assertion (iii) holds.

We end the present section by providing a representation of the conditional expectation $\hat{\zeta}_{t}=\mathbb{E}\left[\zeta_{t} \mid \mathcal{Y}_{t}\right]$ for a $\left(\left(\mathcal{F}_{t}\right), \mathbb{P}\right)$-semimartingale $\zeta=\left(\zeta_{t}, t \in[0, T]\right)$ as the solution of some stochastic differential equation.

$\mathrm{RR} \mathrm{n}^{\circ} 3439$ 
Proposition 2 Let $\zeta=\left(\zeta_{t}, t \in[0, T]\right)$ be a $\left(\left(\mathcal{F}_{t}\right), \mathbb{P}\right)$-semimartingale of the form

$$
\zeta_{t}=\zeta_{0}+\int_{0}^{t} \beta_{s} d s+m_{t}, t \in[0, T]
$$

where $\mathbb{E}\left[\zeta_{0}^{2}\right]<+\infty, \mathbb{E}\left[\int_{0}^{T} \beta_{t}^{2} d t\right]<+\infty$ and $m=\left(m_{t}, t \in[0, T]\right)$ is a square integrable $\left(\left(\mathcal{F}_{t}\right), \mathbb{P}\right)$-martingale such that $<m, N^{*}>_{t}=\int_{0}^{t} \lambda_{s} d<N^{*}>_{s}, t \in$ $[0, T]$. Then the process $\hat{\zeta}=\left(\hat{\zeta}_{t}, t \in[0, T]\right)$ satisfies the stochastic differential equation

$$
\hat{\zeta}_{t}=\hat{\zeta}_{0}+\int_{0}^{t} \hat{\beta}_{s} d s+\int_{0}^{t}\left[\hat{\lambda}_{s}+\zeta_{s} \widehat{q_{s}(X)}-\hat{\zeta}_{s} \pi_{s}(q)\right] d \nu_{s}, t \in[0, T]
$$

where $q(X)$ is the process defined by (32), $\hat{\beta}_{s}=\mathbb{E}\left[\beta_{s} \mid \mathcal{Y}_{s}\right], \hat{\lambda}_{s}=\mathbb{E}\left[\lambda_{s} \mid \mathcal{Y}_{s}\right]$, $\zeta_{s} \widehat{q_{s}(X)}=\mathbb{E}\left[\zeta_{s} q_{s}(X) \mid \mathcal{Y}_{s}\right]$ and $\nu$ is given by (40).

Proof The proof parallels that of Theorem 18.11 in [8]. Define the process $M=\left(M_{t}, t \in[0, T]\right)$ by

$$
M_{t}=\hat{\zeta}_{t}-\hat{\zeta}_{0}-\int_{0}^{t} \hat{\beta}_{s} d s, t \in[0, T]
$$

Then for $0 \leq s \leq t \leq T$ we have

$$
\mathbb{E}\left[M_{t}-M_{s} \mid \mathcal{Y}_{s}\right]=\mathbb{E}\left[\hat{\zeta}_{t}-\hat{\zeta}_{s}-\int_{s}^{t} \hat{\beta}_{u} d u \mid \mathcal{Y}_{s}\right]
$$

However we have

$$
\begin{aligned}
\mathbb{E}\left[\hat{\zeta}_{t}-\hat{\zeta}_{s} \mid \mathcal{Y}_{s}\right] & =\mathbb{E}\left[\zeta_{t}-\zeta_{s} \mid \mathcal{Y}_{s}\right] \\
& =\mathbb{E}\left[\int_{s}^{t} \beta_{u} d u \mid \mathcal{Y}_{s}\right]+\mathbb{E}\left[m_{t}-m_{s} \mid \mathcal{Y}_{s}\right] \\
& =\mathbb{E}\left[\int_{s}^{t} \mathbb{E}\left[\beta_{u} \mid \mathcal{Y}_{u}\right] d u \mid \mathcal{Y}_{s}\right]+\mathbb{E}\left[\mathbb{E}\left[m_{t}-m_{s} \mid \mathcal{F}_{s}\right] \mid \mathcal{Y}_{s}\right] \\
& =\mathbb{E}\left[\int_{s}^{t} \hat{\beta}_{u} d u \mid \mathcal{Y}_{s}\right]
\end{aligned}
$$

because $m$ is a $\left(\left(\mathcal{F}_{t}\right), \mathbb{P}\right)$-martingale. Therefore, $M$ is a locally square integrable $\left(\left(\mathcal{Y}_{t}\right), \mathbb{P}\right)$-martingale. So, by Lemma 3 , there is a $\left(\mathcal{Y}_{t}\right)$-adapted process $\Gamma$ such that $\mathbb{P}$ almost surely

$$
M_{t}=\int_{0}^{t} \Gamma_{s} d \nu_{s}, t \in[0, T]
$$


and we can write

$$
\hat{\zeta}_{t}=\hat{\zeta}_{0}+\int_{0}^{t} \hat{\beta}_{s} d s+\int_{0}^{t} \Gamma_{s} d \nu_{s}, t \in[0, T]
$$

Now we wish to identify the process $\Gamma$. As in the proof of Theorem 8.11 in [8] we shall do it by using the unique decomposition of special semimartingales. From the representation (38) of the process $Z^{*}$ defined by (35), applying the differentiation rule we get

$$
\begin{aligned}
\zeta_{t} Z_{t}^{*}= & \int_{0}^{t} \zeta_{s}\left[q_{s}(X) d<N^{*}>_{s}+d N_{s}^{*}\right]+\int_{0}^{t} Z_{s}^{*}\left[\beta_{s} d s+d m_{s}\right] \\
& +\int_{0}^{t} \lambda_{s} d<N^{*}>_{s}, \quad t \in[0, T] .
\end{aligned}
$$

Proceeding as in [8], one can then derive a decomposition of $\hat{\zeta} Z^{*}$ as a special semimartingale with respect to $\left(\mathcal{Y}_{t}\right)$ where the bounded variation term is

$$
\int_{0}^{t}\left[\zeta_{s} \widehat{q_{s}(X)}+\hat{\lambda}_{s}\right] d<N^{*}>_{s}+\int_{0}^{t} Z_{s}^{*} \hat{\beta}_{s} d s, t \in[0, T]
$$

However, using (40) and (44), applying the differentiation rule we obtain another decomposition of $\hat{\zeta} Z^{*}$ as a special semimartingale with respect to $\left(\mathcal{Y}_{t}\right)$ where the bounded variation term is

$$
\int_{0}^{t}\left[\hat{\zeta}_{s} \pi_{s}(q)+\Gamma_{s}\right] d<N^{*}>_{s}+\int_{0}^{t} Z_{s}^{*} \hat{\beta}_{s} d s, t \in[0, T]
$$

Since the canonical decomposition of the special semimartingale $\hat{\zeta} Z^{*}$ is unique, in particular the integrals in (45) and (46) must be almost surely equal. This gives that $\left\langle N^{*}\right\rangle \otimes \mathbb{P}$ almost everywhere in $[0, T] \times \Omega$ the following holds :

$$
\Gamma_{s}=\hat{\lambda}_{s}+\zeta_{s} \widehat{q_{s}(X)}-\hat{\zeta}_{s} \pi_{s}(q)
$$

Hence, inserting into (44), the representation (43) follows.

Now we may extend partly Zakai's approach (see e.g. $[29,8]$ ) to derive equations for the optimal filter when the signal $X$ is a diffusion process.

$\mathrm{RR} \mathrm{n}^{\circ} 3439$ 


\section{Optimal filtering of a diffusion process in frac- tional Brownian observation noise}

Here we assume that the signal process $X$ satisfies the stochastic differential equation

$$
X_{t}=\eta+\int_{0}^{t} a\left(s, X_{s}\right) d s+\int_{0}^{t} b\left(s, X_{s}\right) d V_{s}^{1 / 2}, t \in[0, T]
$$

where with respect to $\mathbb{P}$ (or $\widetilde{\mathbb{P}}$ ) the process $V^{1 / 2}=\left(V_{t}^{1 / 2}, t \in[0, T]\right.$ ) is a standard Brownian motion independent of $W$ such that the pair $(\eta, \xi)$ is independent of $\left(V^{1 / 2}, W\right)$. Of course we suppose that the distribution $\mu_{(X, \xi)}$ is uniquely well-defined. Moreover we assume that the conditional distribution of $\eta$ given $\xi$ is some fixed $\pi_{0}$.

Let $\phi$ be any smooth function with regular first and second order derivatives $\phi^{\prime}$ and $\phi^{\prime \prime}$ respectively. Then, according to Itô's formula, the process $\left(\phi\left(X_{t}\right), t \in\right.$ $[0, T])$ is a semimartingale with the following decomposition :

$$
\phi\left(X_{t}\right)=\phi(\eta)+\int_{0}^{t} \mathcal{L}_{s} \phi\left(X_{s}\right) d s+\int_{0}^{t} \phi^{\prime}\left(X_{s}\right) b\left(s, X_{s}\right) d V_{s}^{1 / 2}, t \in[0, T],
$$

where, for $s \in[0, T], \mathcal{L}_{s}$ is the second order differential operator defined by

$$
\mathcal{L}_{s} \phi(x)=a(s, x) \phi^{\prime}(x)+\frac{1}{2} b^{2}(s, x) \phi^{\prime \prime}(x), x \in \mathbb{R} .
$$

Hence a straightforward application of Proposition 2 gives an equation for the optimal filter $\pi_{t}$ : for all $t \in[0, T]$,

$$
\pi_{t}(\phi)=\pi_{0}(\phi)+\int_{0}^{t} \pi_{s}\left(\mathcal{L}_{s} \phi\right) d s+\int_{0}^{t}\left[\pi_{s}(\phi q)-\pi_{s}(\phi) \pi_{s}(q)\right] d \nu_{s}
$$

where $\pi_{s}(\phi q)=\mathbb{E}\left[\phi\left(X_{s}\right) q_{s}(X) \mid \mathcal{Y}_{s}\right]$.

In order to be able to derive an analogue of Zakai's formula for the unnormalized filter $\sigma_{t}$, now we deal with the local density process $\Lambda$ in the Girsanov type formula of Proposition 1. Recall that

$$
\Lambda_{t}=\exp \left(\widetilde{N}_{t}-\frac{1}{2}<\widetilde{N}>_{t}\right)
$$


Taking into account equation (22), this definition can be rewritten in terms of the processes $Z$ and $\langle N\rangle$, defined by (34) and (29) respectively, as

$$
\Lambda_{t}=\exp \left(Z_{t}-\frac{1}{2}<N>_{t}\right)
$$

But from the representations (33) and (39) of $\langle N\rangle$ and $Z$ this can also be written as

$$
\Lambda_{t}=\exp \left(\int_{0}^{t} q_{s}(X) d Z_{s}^{*}-\frac{1}{2} \int_{0}^{t} q_{s}^{2}(X) d<N^{*}>_{s}\right)
$$

Hence $\Lambda$ appears as the solution of the stochastic differential equation

$$
\Lambda_{t}=1+\int_{0}^{t} q_{s}(X) \Lambda_{s} d Z_{s}^{*}, t \in[0, T]
$$

Recall that by definition of the unnormalized filter $\sigma_{t}$

$$
\sigma_{t}(\phi)=\tilde{\mathbb{E}}\left[\phi\left(X_{t}\right) \Lambda_{t} \mid \mathcal{Y}_{t}\right]
$$

and in particular

$$
\widetilde{\Lambda}_{t} \triangleq \sigma_{t}(1)=\widetilde{\mathbb{E}}\left[\Lambda_{t} \mid \mathcal{Y}_{t}\right]
$$

Now we obtain a formula for the process $\widetilde{\Lambda}=\left(\widetilde{\Lambda}_{t}, t \in[0, T]\right)$ :

Proposition 3 The process $\widetilde{\Lambda}$ defined by (50) is given $\mathbb{P}$ almost surely by

$$
\widetilde{\Lambda}_{t}=\exp \left\{\int_{0}^{t} \pi_{s}(q) d Z_{s}^{*}-\frac{1}{2} \int_{0}^{t} \pi_{s}^{2}(q) d<N^{*}>_{s}\right\}, t \in[0, T]
$$

where $Z^{*}$ is defined by (35) and $\pi_{s}(q)$ is the conditional expectation $\pi_{s}(q)=$ $\mathbb{E}\left[q_{s}(X) \mid \mathcal{Y}_{s}\right]$ with respect to $\mathbb{P}$.

Proof The proof parallels that of Theorem 18.21 in [8]. Recall that $\Lambda$ is a $\left(\left(\mathcal{F}_{t}\right), \widetilde{\mathbb{P}}\right)$-martingale. Of course, consequently $\widetilde{\Lambda}$ is a $\left(\left(\mathcal{Y}_{t}\right), \widetilde{\mathbb{P}}\right)$-martingale.

Under $\widetilde{\mathbb{P}}$, the process $Z^{*}$ which can be written as

$$
Z_{t}^{*}=\int_{0}^{t} k_{*}^{t}(s) d \widetilde{W}_{s}, t \in[0, T]
$$

$\mathrm{RR} \mathrm{n}^{\circ} 3439$ 
is a continuous Gaussian $\left(\mathcal{Y}_{t}\right)$-martingale such that $\left.\left\langle Z^{*}\right\rangle=<N^{*}\right\rangle$. Hence there exists a $\left(\mathcal{Y}_{t}\right)$-adapted process $g$ such that $\widetilde{\mathbb{P}}$ almost surely

$$
\widetilde{\Lambda}_{t}=1+\int_{0}^{t} g_{s} d Z_{s}^{*}, t \in[0, T]
$$

We shall identify $g$ by using the unique decomposition of special semimartingales. Using the differentiation rule to compute $\Lambda Z^{*}$, from (49) and (38) we get

$$
\begin{aligned}
\Lambda_{t} Z_{t}^{*} & =\int_{0}^{t} \Lambda_{s} d Z_{s}^{*}+\int_{0}^{t} Z_{s}^{*} d \Lambda_{s}+<\Lambda, Z^{*}>_{t} \\
& =\int_{0}^{t} \Lambda_{s} d Z_{s}^{*}+\int_{0}^{t} Z_{s}^{*} q_{s}(X) \Lambda_{s} d Z_{s}^{*}+\int_{0}^{t} q_{s}(X) \Lambda_{s} d<N^{*}>_{s}
\end{aligned}
$$

Proceeding as in the proof of Theorem 18.21 in [8] one can derive a decomposition of $\widetilde{\Lambda} Z^{*}$ as a special $\left(\left(\mathcal{Y}_{t}\right), \widetilde{\mathbb{P}}\right)$-semimartingale where the bounded variation term is

$$
\int_{0}^{t} \Lambda_{s} \widetilde{q_{s}}(X) d<N^{*}>_{s}, t \in[0, T]
$$

where $\Lambda_{s} \widetilde{q_{s}(X)}=\widetilde{\mathbb{E}}\left[\Lambda_{s} q_{s}(X) \mid \mathcal{Y}_{s}\right]$.

However, using (52) and (40), again applying the differentiation rule we can obtain another decomposition of $\widetilde{\Lambda} Z^{*}$ as a special $\left(\left(\mathcal{Y}_{t}\right), \widetilde{\mathbb{P}}\right)$-semimartingale where the bounded variation term is

$$
\int_{0}^{t} g_{s} d<N^{*}>_{s}, t \in[0, T]
$$

By the uniqueness of the decomposition of a special semimartingale we obtain that $\left\langle N^{*}>\otimes \widetilde{\mathbb{P}}\right.$ almost everywhere in $[0, T] \times \Omega$

$$
\begin{aligned}
g_{s} & =\widetilde{\Lambda_{s} q_{s}(X)} \\
& =\widetilde{\mathbb{E}}\left[\Lambda_{s} q_{s}(X) \mid \mathcal{Y}_{s}\right]
\end{aligned}
$$

However, from (28) and (50), this means that $\left\langle N^{*}>\otimes \widetilde{\mathbb{P}}\right.$ almost everywhere in $[0, T] \times \Omega$

$$
g_{s}=\widetilde{\Lambda}_{s} \pi_{s}(q)
$$


Substituting in (52), we obtain the equation

$$
\widetilde{\Lambda}_{t}=1+\int_{0}^{t} \widetilde{\Lambda}_{s} \pi_{s}(q) d Z_{s}^{*}, t \in[0, T]
$$

which has, with respect to $\widetilde{\mathbb{P}}$ the unique solution

$$
\widetilde{\Lambda}_{t}=\exp \left\{\int_{0}^{t} \pi_{s}(q) d Z_{s}^{*}-\frac{1}{2} \int_{0}^{t} \pi_{s}^{2}(q) d<N^{*}>_{s}\right\}, t \in[0, T]
$$

Hence, since $\mathbb{P}$ is equivalent to $\widetilde{\mathbb{P}}$, the proposition is proved.

Now we obtain an analogue of classical Zakai's equation (see e.g. [29, 8]) for the unnormalized filter $\sigma_{t}$ :

Proposition 4 Let $\sigma_{t}$ be the unnormalized filter in the signal-observation model defined by equations (47) and (1). Then for any smooth function $\phi$ the following equation holds :

$$
\sigma_{t}(\phi)=\sigma_{0}(\phi)+\int_{0}^{t} \sigma_{s}\left(\mathcal{L}_{s} \phi\right) d s+\int_{0}^{t} \sigma_{s}(\phi q) d Z_{s}^{*}, t \in[0, T]
$$

where the process $Z^{*}$ is given by (35) and $\sigma_{0}(\phi)=\pi_{0}(\phi)$.

Proof We observe that from its definition we have

$$
\sigma_{t}(\phi)=\widetilde{\Lambda}_{t} \pi_{t}(\phi)
$$

But, from equations (48), (53) and (40) we have

$$
\pi_{t}(\phi)=\pi_{0}(\phi)+\int_{0}^{t} \pi_{s}\left(\mathcal{L}_{s} \phi\right) d s+\int_{0}^{t}\left[\pi_{s}(\phi q)-\pi_{s}(\phi) \pi_{s}(q)\right] d \nu_{s}, t \in[0, T]
$$

and

$$
\widetilde{\Lambda}_{t}=1+\int_{0}^{t} \widetilde{\Lambda}_{s} \pi_{s}^{2}(q) d<N^{*}>_{s}+\int_{0}^{t} \widetilde{\Lambda}_{s} \pi_{s}(q) d \nu_{s}, t \in[0, T]
$$

Then, applying Itô's formula, we get

$$
d \sigma_{t}(\phi)=\widetilde{\Lambda}_{t} d \pi_{t}(\phi)+\pi_{t}(\phi) d \widetilde{\Lambda}_{t}+d<\widetilde{\Lambda}, \pi(\phi)>_{t}
$$

$\mathrm{RR} \mathrm{n}^{\circ} 3439$ 


$$
\begin{aligned}
= & \widetilde{\Lambda}_{t} \pi_{t}\left(\mathcal{L}_{t} \phi\right) d t+\widetilde{\Lambda}_{t}\left[\pi_{t}(\phi q)-\pi_{t}(\phi) \pi_{t}(q)\right] d \nu_{t} \\
& +\pi_{t}(\phi) \widetilde{\Lambda}_{t} \pi_{t}(q) d \nu_{t}+\pi_{t}(\phi) \widetilde{\Lambda}_{t} \pi_{t}^{2}(q) d<N^{*}>_{t} \\
+ & \widetilde{\Lambda}_{t} \pi_{t}(q)\left[\pi_{t}(\phi q)-\pi_{t}(\phi) \pi_{t}(q)\right] d<N^{*}>_{t} \\
= & \widetilde{\Lambda}_{t} \pi_{t}\left(\mathcal{L}_{t} \phi\right) d t+\widetilde{\Lambda}_{t} \pi_{t}(\phi q)\left[d \nu_{t}+\pi_{t}(q) d<N^{*}>_{t}\right] .
\end{aligned}
$$

Again, using (55) and (40), the equation can be rewritten as

$$
d \sigma_{t}(\phi)=\sigma_{t}\left(\mathcal{L}_{t} \phi\right) d t+\sigma_{t}(\phi q) d Z_{t}^{*},
$$

which gives nothing but (54).

Actually, from Propositions 3 and 4, it appears that to give a complete solution to the filtering problem we need also an equation for the conditional expectation $\pi_{t}(\psi)$ of a functional $\psi\left(X^{(t)}\right)$ of the state process, if only for $\pi_{t}(q)$. In the next section we investigate this question in the case of linear systems for which we show that closed form equations can be derived for the optimal filter.

\section{Optimal filtering in conditionally Gaussian li- near systems with fractional Brownian obser- vation noise}

Let us deal here with the particular situation where the dynamics of the process $(X, Y)$ is linear. Precisely we suppose that, written in differential notation, under $\mathbb{P}$ it is given by

$$
\left\{\begin{array}{l}
d X_{t}=a(t) X_{t} d t+b(t) d V_{t}^{1 / 2}, t \in[0, T], X_{0}=\eta \\
d Y_{t}=A(t) X_{t} d t+B(t) d W_{t}, t \in[0, T], Y_{0}=\xi
\end{array}\right.
$$

We assume that the random initial conditions $\eta$ and $\xi$ in (1) are such that the pair $(\eta, \xi)$ is independent of $\left(V^{1 / 2}, W\right)$ where $V^{1 / 2}$ and $W$ are independent Brownian motion and $\mathrm{fBm}$ with Hurst parameter $H$ respectively. The conditional distribution $\pi_{0}$ is assumed to be Gaussian with mean $\hat{\eta}_{0}=\hat{\eta}_{0}(\xi)$ and 
variance $\gamma_{0}=\gamma_{0}(\xi)$ which is supposed almost surely positive. Clearly, under these assumptions, the distribution of process $(X, Y)$ is uniquely well-defined and enters into the general framework described above. Moreover it is obvious that the pair $(X, Y)$ is conditionally Gaussian i.e. for all $t \in[0, T], n=1,2, \ldots$ and $t_{1}, t_{2}, \ldots, t_{n} \in[0, t]$ the joint conditional distribution of $\left(X_{t_{1}}, X_{t_{2}}, \ldots, X_{t_{n}}\right)$ given $\mathcal{Y}_{t}$ is Gaussian.

Notice that here, for a continuous function $x=\left(x_{t}, t \in[0, T]\right)$, according to definition (15), we have $C(x)(t)=C(t) x(t)$ where $C(t)=B^{-1}(t) A(t)$ for all $t \in[0, T]$. Hence, since moreover the functional $C \rightarrow\left(q_{t_{1}}^{C}, q_{t_{2}}^{C}, \ldots, q_{t_{n}}^{C}\right)$ defined from (12) and (10) is linear, the system $((X, q(X)), Y)$ is also conditionally Gaussian.

Then in particular for any $t \in[0, T]$ the optimal filter $\pi_{t}$ is a Gaussian distribution and the filtering equations can be reduced to equations for its mean $\hat{X}_{t}$ and for the conditional variance $\gamma(t)$ of the filtering error i.e.

$$
\hat{X}_{t} \triangleq \mathbb{E}\left[X_{t} \mid \mathcal{Y}_{t}\right] ; \gamma(t) \triangleq \mathbb{E}\left[\left(X_{t}-\hat{X}_{t}\right)^{2} \mid \mathcal{Y}_{t}\right], t \in[0, T]
$$

We shall show that here a closed form system of equations for $\hat{X}_{t}$ and $\gamma_{t}$ can be determined. We introduce the process $\gamma_{X, q}$ defined as

$$
\gamma_{X, q}(t) \triangleq \mathbb{E}\left[\left(X_{t}-\hat{X}_{t}\right)\left(q_{t}(X)-\pi_{t}(q)\right) \mid \mathcal{Y}_{t}\right], t \in[0, T]
$$

Now we start with the following :

Lemma 4 For all $t \in[0, T]$, the mean $\hat{X}_{t}$ and the variance $\gamma(t)$ of the optimal filter $\pi_{t}$ in model (56) satisfy the equations

$$
\hat{X}_{t}=\hat{\eta}_{0}+\int_{0}^{t} a(s) \hat{X}_{s} d s+\int_{0}^{t} \gamma_{X, q}(s) d \nu_{s}
$$

and

$$
\gamma(t)=\gamma_{0}+\int_{0}^{t} 2 a(s) \gamma(s) d s+\int_{0}^{t} b^{2}(s) d s-\int_{0}^{t} \gamma_{X, q}^{2}(s) d<N^{*}>_{s}
$$

where $\gamma_{X, q}$ is the process given by (58).

$\mathrm{RR} \mathrm{n}^{\circ} 3439$ 
Proof Applying Proposition 2 to the semimartingale $X$ we obtain

$$
\left.\hat{X}_{t}=\hat{\eta}_{0}+\int_{0}^{t} a(s) \hat{X}_{s} d s+\int_{0}^{t}\left[X_{s} \widehat{q_{s}}(X)-\widehat{X_{s}} \pi_{s}(q)\right)\right] d \nu_{s}, t \in[0, T] .
$$

Therefore equation (59) holds since the process appearing in the martingale part of the right hand side of this representation is noting but $\gamma_{X, q}$ given by (58). Now applying Itô's formula, we get the following representation of the semimartingale $\hat{X}^{2}$ :

$$
\begin{aligned}
{\left[\hat{X}_{t}\right]^{2}=\left[\hat{\eta}_{0}\right]^{2} } & +\int_{0}^{t} 2 a(s)\left[\hat{X}_{s}\right]^{2} d s+\int_{0}^{t} \gamma_{X, q}^{2}(s) d<N^{*}>_{s} \\
& +\int_{0}^{t} 2 \gamma_{X, q}(s) \hat{X}_{s} d \nu_{s}, t \in[0, T] .
\end{aligned}
$$

On the other hand, applying Itô's formula to the semimartingale $X^{2}$ we get

$$
X_{t}^{2}=\eta^{2}+\int_{0}^{t} 2 a(s) X_{s}^{2} d s+\int_{0}^{t} b^{2}(s) d s+\int_{0}^{t} 2 b(s) X_{s} d V_{s}^{1 / 2}, t \in[0, T]
$$

Hence, again applying Proposition 2, we obtain

$$
\begin{aligned}
\widehat{X_{t}^{2}}=\widehat{\eta^{2}}{ }_{0} & +\int_{0}^{t} 2 a(s) \widehat{X_{s}^{2}} d s+\int_{0}^{t} b^{2}(s) d s \\
& +\int_{0}^{t}\left[X_{s}^{2} \widehat{q_{s}(X)}-\widehat{X_{s}^{2}} \pi_{s}(q)\right] d \nu_{s}, \quad, t \in[0, T] .
\end{aligned}
$$

Subtracting equation (61) from equation (62) leads to

$$
\begin{aligned}
\gamma(t)= & \gamma_{0}+\int_{0}^{t} 2 a(s) \gamma(s) d s+\int_{0}^{t} b^{2}(s) d s-\int_{0}^{t} \gamma_{X, q}^{2}(s) d<N^{*}>_{s} \\
& +\int_{0}^{t}\left[X_{s}^{2} \widehat{q_{s}}(X)-\widehat{X_{s}^{2}} \pi_{s}(q)-2 \gamma_{X, q}(s) \hat{X}_{s}\right] d \nu_{s}, t \in[0, T] .
\end{aligned}
$$

But, using the conditionally Gaussian property, it is readily seen that the process appearing as the integrand in the martingale part of the right hand side above is almost surely zero. Consequently the last equation is nothing but (60). 
Remark 3 Let us emphasize that in the case when the observation noise is also a standard Brownian motion, i.e. $H=\frac{1}{2}$, then the previous optimal filtering equations reduce to the usual Kalman equations. Actually, taking into account Remarks $1(\mathrm{~d})$ and $2(\mathrm{c})$, in this case we have

$$
q_{t}(X)=C(t) X_{t} ; \pi_{t}(q)=C(t) \hat{X}_{t} ; \gamma_{X, q}(t)=C(t) \gamma(t), t \in[0, T]
$$

Hence from (40) we have also

$$
d \nu_{t}=B^{-1}(t) d Y_{t}-C(t) \hat{X}_{t} d t=B^{-1}(t)\left[d Y_{t}-A(t) \hat{X}_{t} d t\right] .
$$

Then equations (59)-(60) in Lemma 4 become

$$
\begin{gathered}
\hat{X}_{t}=\hat{\eta}_{0}+\int_{0}^{t} a(s) \hat{X}_{s} d s+\int_{0}^{t} \frac{A(s)}{B^{2}(s)} \gamma(s)\left[d Y_{s}-A(s) \hat{X}_{s} d s\right], t \in[0, T], \\
\gamma(t)=\gamma_{0}+\int_{0}^{t} 2 a(s) \gamma(s) d s+\int_{0}^{t} b^{2}(s) d s-\int_{0}^{t} \frac{A^{2}(s)}{B^{2}(s)} \gamma^{2}(s) d s, t \in[0, T],
\end{gathered}
$$

which are nothing but the classical Kalman filter equations for a linear system driven by Brownian noises (see e.g. [17]).

Actually Lemma 4 permits to give an explicit solution of the filtering problem in the specific case of a noise free state equation when actually the signal is a fixed random variable i.e. in (56) the functions $a$ and $b$ are identically zero and hence $X_{t} \equiv \eta$. This means that the observation process is $Y$ where

$$
Y_{t}=\xi+\int_{0}^{t} A(s) \eta d s+\int_{0}^{t} B(s) d W_{s}, \quad t \in[0, T]
$$

We shall use the notation $\hat{\eta}_{t}=\mathbb{E}\left[\eta \mid \mathcal{Y}_{t}\right]$ and then $\gamma(t)=\mathbb{E}\left[\left(\eta-\hat{\eta}_{t}\right)^{2} \mid \mathcal{Y}_{t}\right]$. Here of course the process $q(X)$ defined by (32) and the corresponding conditional expectation $\pi_{t}(q)$ reduce to

$$
q_{t}(X)=q_{t}^{C} \eta ; \pi_{t}(q)=q_{t}^{C} \hat{\eta}_{t}, t \in[0, T]
$$

where $q^{C}$ is given by (12). Hence also

$$
\gamma_{X, q}(t)=q_{t}^{C} \gamma(t), t \in[0, T],
$$

$\mathrm{RR} \mathrm{n}^{\circ} 3439$ 
and equation (60) becomes

$$
\gamma(t)=\gamma_{0}-\int_{0}^{t} \gamma^{2}(s)\left[q_{s}^{C}\right]^{2} d<N^{*}>_{s}, t \in[0, T] .
$$

This equation has an explicit solution

$$
\gamma(t)=\left(\gamma_{0}^{-1}+\int_{0}^{t}\left[q_{s}^{C}\right]^{2} d<N^{*}>_{s}\right)^{-1}, t \in[0, T] .
$$

Observe that from (6) and (11) it can be rewritten as

$$
\gamma(t)=\left(\gamma_{0}^{-1}+\int_{0}^{t} k_{C}^{t}(s) C(s) d s\right)^{-1}, t \in[0, T] .
$$

Moreover equation (59) reduces to

$$
\hat{\eta}_{t}=\hat{\eta}_{0}+\int_{0}^{t} q_{s}^{C} \gamma(s) d \nu_{s}, t \in[0, T]
$$

i.e., taking into account the representation (40) of the innovation process $\nu$,

$$
\hat{\eta}_{t}=\hat{\eta}_{0}+\int_{0}^{t} q_{s}^{C} \gamma(s)\left[d Z_{s}^{*}-q_{s}^{C} \hat{\eta}_{s} d<N^{*}>_{s}\right],, t \in[0, T] .
$$

Then the conditional mean $\hat{\eta}_{t}$ can be obtained computing

$$
\begin{aligned}
d\left[\gamma_{t}^{-1} \hat{\eta}_{t}\right] & =\hat{\eta}_{t} d \gamma^{-1}(t)+\gamma^{-1}(t) d \hat{\eta}_{t} \\
& =\hat{\eta}_{t}\left[q_{t}^{C}\right]^{2} d<N^{*}>_{t}+\gamma^{-1}(t) \gamma(t) q_{t}^{C}\left[d Z_{t}^{*}-q_{t}^{C} \hat{\eta}_{t} d<N^{*}>_{t}\right] \\
& =q_{t}^{C} d Z_{t}^{*}
\end{aligned}
$$

This gives

$$
\begin{aligned}
\gamma_{t}^{-1} \hat{\eta}_{t} & =\gamma_{0}^{-1} \hat{\eta}_{0}+\int_{0}^{t} q_{s}^{C} d Z_{s}^{*} \\
& =\gamma_{0}^{-1} \hat{\eta}_{0}+\int_{0}^{t} k_{C}^{t}(s) B^{-1}(s) d Y_{s}, \operatorname{tin}[0, T]
\end{aligned}
$$

where the last equality can be easily checked from (38) and $d Y_{s}=A(s) \eta d s+$ $B(s) d W_{s}$.

Finally, this leads to

$$
\hat{\eta}_{t}=\gamma(t)\left(\gamma_{0}^{-1} \hat{\eta}_{0}+\int_{0}^{t} k_{C}^{t}(s) B^{-1}(s) d Y_{s}\right)
$$


Of course it appears that $\left(\hat{\eta}_{t}, \gamma(t)\right)$ is nothing but the solution of the filtering problem which was directly derived for this simple model in [16] (see also [15]). Observe also that this is an analogue for fBm observation noise of Theorem 12.2 in [17] for Brownian motion noise.

Example Let us consider the particular case where $A=B \equiv 1$ (and then also $C \equiv 1)$ i.e. we deal with the model $Y_{t}=\xi+\eta t+W_{t}, \quad t \in[0, T]$. Here we get

$$
\hat{\eta}_{t}=\gamma_{t}\left\{\gamma_{0}^{-1} \hat{\eta}_{0}+\kappa_{H}^{-1} \int_{0}^{t} s^{\frac{1}{2}-H}(t-s)^{\frac{1}{2}-H} d Y_{s}\right\}
$$

and

$$
\gamma(t)=\left\{\gamma_{0}^{-1}+\lambda_{H}^{-1} t^{2-2 H}\right\}^{-1}
$$

Now let us come back to the more general case of this section. We look for equations for the processes $\pi(q)$ and $\gamma_{X, q}$. We begin by establishing a representation of the process $q(X)$ as the solution of an Itô-Volterra type equation with deterministic coefficients.

Lemma 5 Let the process $q(X)=\left(q_{t}(X), t \in[0, T]\right)$ be defined by (31)-(32) with $C(x)(t)=C(t) x(t), C(t)=B^{-1}(t) A(t), t \in[0, T]$. Then $q(X)$ satisfies the following equation

$$
\begin{aligned}
q_{t}(X)= & q^{C}(t, 0) \eta+\int_{0}^{t} a(u) q^{C}(t, u) X_{u} d u \\
& +\int_{0}^{t} b(u) q^{C}(t, u) d V_{u}^{1 / 2}, t \in[0, T],
\end{aligned}
$$

where $q^{C}(.,$.$) permits the representation (14).$

Proof Starting from (13) and the first equation in (56), for all $t \in[0, T]$ we can write

$$
\begin{aligned}
\int_{0}^{t} q_{s}(X) d<N^{*}>_{s}= & \int_{0}^{t} k_{*}^{t}(s) C(s) X_{s} d s \\
= & \int_{0}^{t} k_{*}^{t}(s) C(s)\left\{\eta+\int_{0}^{s} a(u) X_{u} d u+\int_{0}^{s} b(u) d V_{u}^{1 / 2}\right\} d s \\
= & {\left[\int_{0}^{t} k_{*}^{t}(s) C(s) d s\right] \eta+\int_{0}^{t} a(u)\left\{\int_{u}^{t} k_{*}^{t}(s) C(s) d s\right\} X_{u} d u } \\
& \quad+\int_{0}^{t} b(u)\left\{\int_{u}^{t} k_{*}^{t}(s) C(s) d s\right\} d V_{u}^{1 / 2}
\end{aligned}
$$

$\mathrm{RR} \mathrm{n}^{\circ} 3439$ 
Hence, taking into account representation (14), this can be rewritten as

$$
\begin{aligned}
\int_{0}^{t} q_{s}(X) d<N^{*}>_{s}=\left[\int_{0}^{t}\right. & \left.q^{C}(s, 0) d<N^{*}>_{s}\right] \eta \\
& +\int_{0}^{t} a(u)\left\{\int_{u}^{t} q^{C}(s, u) d<N^{*}>_{s}\right\} X_{u} d u \\
& +\int_{0}^{t} b(u)\left\{\int_{u}^{t} q^{C}(s, u) d<N^{*}>_{s}\right\} d V_{u}^{1 / 2} .
\end{aligned}
$$

Finally we get

$$
\begin{aligned}
\int_{0}^{t} q_{s}(X) d<N^{*}>_{s}= & \int_{0}^{t} \\
& q^{C}(s, 0) \eta d<N^{*}>_{s} \\
& +\int_{0}^{t}\left\{\int_{0}^{s} a(u) q^{C}(s, u) X_{u} d u\right\} d<N^{*}>_{s} \\
& +\int_{0}^{t}\left\{\int_{0}^{s} b(u) q^{C}(s, u) d V_{u}^{1 / 2}\right\} d<N^{*}>_{s}
\end{aligned}
$$

which means nothing but that representation (63) holds.

Of course $q(X)$ is not a semimartingale and we can not directly apply Proposition 2 to obtain an equation for the process $\pi(q)$. To overcome this difficulty we shall use the technique which was proposed in $[12,13,14]$ to transform the problem into a filtering problem concerning a semimartingale. Let us fix some $t \in[0, T]$ and define the process $q^{t}(X)=\left(q_{s}^{t}(X), s \in[0, t]\right)$ by

$$
\begin{aligned}
q_{s}^{t}(X)= & q^{C}(t, 0) \eta+\int_{0}^{s} a(u) q^{C}(t, u) X_{u} d u \\
& +\int_{0}^{s} b(u) q^{C}(t, u) d V_{u}^{1 / 2}, s \in[0, t] .
\end{aligned}
$$

Observe that it is a semimartingale and moreover, taking $s=t$ in (64), from (63) we get

$$
q_{t}^{t}(X)=q_{t}(X)
$$

We introduce the processes $\gamma_{X, q}(t,$.$) and \gamma_{q, q}(t,$.$) by$

$$
\begin{gathered}
\gamma_{X, q}(t, s) \triangleq \mathbb{E}\left[\left(X_{s}-\hat{X}_{s}\right)\left(q_{s}^{t}(X)-\pi_{s}\left(q^{t}\right)\right) \mid \mathcal{Y}_{s}\right], 0 \leq s \leq t, \\
\gamma_{q, q}(t, s) \triangleq \mathbb{E}\left[\left(q_{s}^{t}(X)-\pi_{s}\left(q^{t}\right)\right)\left(q_{s}(X)-\pi_{s}(q)\right) \mid \mathcal{Y}_{s}\right], 0 \leq s \leq t .
\end{gathered}
$$


Of course, taking $s=t$ in these definitions, it appears that $\gamma_{X, q}(t, t)$ is nothing but $\gamma_{X, q}(t)$ defined by $(58)$ and $\gamma_{q, q}(t, t)$ is the conditional variance $\mathbb{E}\left[\left(q_{t}(X)-\right.\right.$ $\left.\left.\pi_{t}(q)\right)^{2} \mid \mathcal{Y}_{t}\right]$

Then we can state

Lemma 6 Let the process $q^{t}(X)$ be defined by (64). Then the following representations of the conditional expectation $\pi_{s}\left(q^{t}\right)=\mathbb{E}\left[q_{s}^{t}(X) \mid \mathcal{Y}_{s}\right]$ and of the conditional covariances $\gamma_{X, q}(t, s)$ and $\gamma_{q, q}(t, s)$, defined by (66) and (67) respectively, hold for $0 \leq s \leq t$ :

$$
\begin{aligned}
\pi_{s}\left(q^{t}\right)= & q^{C}(t, 0) \hat{\eta}_{0}+\int_{0}^{s} a(u) q^{C}(t, u) \hat{X}_{u} d u+\int_{0}^{s} \gamma_{q, q}(t, u) d \nu_{u} \\
\gamma_{X, q}(t, s)= & q^{C}(t, 0) \gamma_{0}+\int_{0}^{s} a(u)\left[q^{C}(t, u) \gamma(u)+\gamma_{X, q}(t, u)\right] d u \\
& +\int_{0}^{s} b^{2}(u) q^{C}(t, u) d u-\int_{0}^{s} \gamma_{X, q}(u) \gamma_{q, q}(t, u) d<N^{*}>_{u},
\end{aligned}
$$

$\gamma_{q, q}(t, s)=q^{C}(t, 0) q^{C}(s, 0) \gamma_{0}$

$$
\begin{aligned}
& +\int_{0}^{s} a(u)\left[q^{C}(t, u) \gamma_{X, q}(s, u)+q^{C}(s, u) \gamma_{X, q}(t, u)\right] d u \\
& +\int_{0}^{s} b^{2}(u) q^{C}(t, u) q^{C}(s, u) d u-\int_{0}^{s} \gamma_{q, q}(t, u) \gamma_{q, q}(s, u) d<N^{*}>_{u} .
\end{aligned}
$$

Proof Equation (68) is an immediate consequence of Proposition 2 applied to the semimartingale $q^{t}(X)$.

To get (69) we start from the first equation in (56) and equation (64) to write the semimartingale representation of $X q^{t}(X)$ by using the differentiation rule:

$$
\begin{aligned}
X_{s} q_{s}^{t}(X)= & q^{C}(t, 0) \eta^{2}+\int_{0}^{s} a(u)\left[q^{C}(t, u) X_{u}^{2}+X_{u} q_{u}^{t}(X)\right] d u \\
& \quad+\int_{0}^{s} b^{2}(u) q^{C}(t, u) d u+\int_{0}^{s} b(u)\left[q^{C}(t, u) X_{u}+q_{u}^{t}(X)\right] d V_{u}^{1 / 2}
\end{aligned}
$$

Hence, again applying Proposition 2, we get

$$
\begin{gathered}
X_{s} \widehat{q_{s}^{t}(X)}=q^{C}(t, 0) \widehat{\eta^{2}}{ }_{0}+\int_{0}^{s} a(u)\left[q^{C}(t, u) \widehat{X_{u}^{2}}+X_{u} \widehat{q_{u}^{t}}(X)\right] d u \\
+\int_{0}^{s} b^{2}(u) q^{C}(t, u) d u+\int_{0}^{s}\left[X_{u} q_{u}^{t}\left(\widehat{X)} q_{u}(X)-X_{u} \widehat{q_{u}^{t}}(X) \pi_{u}(q)\right] d \nu_{u} .\right.
\end{gathered}
$$

$\mathrm{RR} \mathrm{n}^{\circ} 3439$ 
Now, from (59) and (68), we get a representation of the semimartingale $\hat{X} \pi\left(q^{t}\right)$ :

$$
\begin{aligned}
\widehat{X}_{s} \pi_{s}\left(q^{t}\right)= & q^{C}(t, 0)\left[\widehat{\eta}_{0}\right]^{2}+\int_{0}^{s} a(u)\left[q^{C}(t, u){\widehat{X_{u}}}^{2}+\widehat{X}_{u} \pi_{u}\left(q^{t}\right)\right] d u \\
& +\int_{0}^{s} \gamma_{X, q}(u) \gamma_{q, q}(t, u) d<N^{*}>_{u} \\
& +\int_{0}^{s}\left[\widehat{X_{u}} \gamma_{q, q}(t, u)+\gamma_{X, q}(t, u) \pi_{u}\left(q^{t}\right)\right] d \nu_{u} .
\end{aligned}
$$

Subtracting (72) from equation (71) leads immediately to (69) since, from the conditionally Gaussian property, it appears that the martingale part of the obtained semimartingale is zero.

Equation (70) can be obtained, through developments quite parallel to those in the previous proofs, by computing $\pi_{s}\left(q^{t} q^{r}\right)-\pi_{s}\left(q^{t}\right) \pi_{s}\left(q^{r}\right)$ for $r, t \geq s$ and observing that, due to $(65), \gamma_{q, q}(t, s)$ is nothing but the value of this quantity for $r=s$.

Now, as an immediate consequence of Lemmas 4,5 and 6, we may give the solution of the filtering problem in the linear system (56):

Proposition 5 Let $(X, Y)$ the solution process of the linear system (56). Let $q(X)=\left(q_{t}(X), t \in[0, T]\right)$ be the auxiliary process defined by (31)-(32) with $C(x)(t)=C(t) x(t), C(t)=B^{-1}(t) A(t), t \in[0, T]$. Let also $q^{C}(.,$.$) be the$ function permitting the representation (14). Then the mean $\hat{X}_{t}$ and the variance $\gamma(t)$ of the optimal filter $\pi_{t}$ of $X$ satisfy the equations (59) and (60) where

$$
\begin{gathered}
\nu_{t}=\int_{0}^{t} k_{*}^{t}(s) B^{-1}(s) d Y_{s}-\int_{0}^{t} \pi_{s}(q) d<N^{*}>_{s}, t \in[0, T] \\
\pi_{t}(q)=q^{C}(t, 0) \hat{\eta}_{0}+\int_{0}^{t} a(u) q^{C}(t, u) \hat{X}_{u} d u+\int_{0}^{t} \gamma_{q, q}(t, u) d \nu_{u},
\end{gathered}
$$

and processes $\gamma_{X, q}(.,$.$) and \gamma_{q, q}(.,$.$) satisfy (69) and (70) respectively.$

Remark 4 Let us mention that in the case when the noise in the state equation is also a $\mathrm{fBm}$, developments similar to those above are still possible. In that case one may follow for the signal $X$ the technique, which has been used above for $q(X)$, to transform the original problem into a filtering problem concerning a semimartingale. This will be reported in a forthcoming paper as well as extensions to nonlinear systems. 


\section{References}

[1] Beran, J. : Statistical models for data with long-range dependence, Statistical Science, 7, 1992, pp. 404-427.

[2] Beran, J. : Statistics for Long-Memory Processes, Chapman \& Hall, New York, 1994.

[3] Comte, F. : Simulation and estimation of long memory continuous time models, Journal of Time Series Analysis, 17, (1) 1994, pp. 19-36.

[4] Coelho, R. and Decreusefond, L. : Video correlated traffic models for broadband communications networks, In: Proceedings of the ITC Seminar on Teletraffic Management, 17, (1), 1995, pp. 19-36.

[5] Coutin, L. and Decreusefond, L. :Filtering theory for solutions of differential equations driven by a fractional Brownian motion, Preprint, 1997.

[6] Dai, W. and Heyde, C.C. : Itô's formula with respect to fractional Brownian motion and its application, Journal of Applied Math. and Stochastic Analysis, 9, (4), 1996, pp. 439-448.

[7] Decreusefond, L. and Üstünel, A.S. : Stochastic analysis of the fractional Brownian motion, Potential Analysis, to appear, 1998.

[8] Elliott, R.J.: Stochastic Calculus and Applications, Springer-Verlag, New York, 1982.

[9] Gripenberg, G. and Norros, I. : On the prediction of fractional Brownian motion, Journal of Applied Probability, 33, 1997, pp. 400-410.

[10] Hurst, H.E. : Long-term storage capacity of reservoirs, Trans. Amer. Soc. Civil Eng., 116, 1951, pp. 400-410.

[11] Hurst, H.E. : Methods of using long-term storage in reservoirs, Proc. Inst. Civil Engineers, Part I, Chapter 5, 1956, pp. 519-590.

[12] Kleptsyna, M.L., Kloeden, P.E. and Ahn, V.V. : Linear filtering with fractional Brownian motion, Stochastic Analysis and its Applications, to appear, 1998.

$\mathrm{RR} \mathrm{n}^{\circ} 3439$ 
[13] Kleptsyna, M.L., Kloeden, P.E. and Ahn, V.V. : Nonlinear filtering with fractional Brownian motion, Information Transmission Problems, to appear, 1998.

[14] Kleptsyna, M.L., Kloeden, P.E. and Ahn, V.V. : Linear filtering with fractional Brownian motion in the signal and observation processes, Journal of Applied Math. and Stochastic Analysis, to appear, 1998.

[15] Le Breton, A. : Filtering and parameter estimation in a simple linear model driven by a fractional Brownian motion, Statistics and Probability Letters, to appear, 1998.

[16] Le Breton, A. : Une approche de type Girsanov pour le filtrage dans un système linéaire simple avec bruit brownien fractionnaire, C.R. Acad. Sci. Paris, t.326, Serie I, 1998, pp. 997-1002.

[17] Liptser, R. S. and Shiryaev, A. N. : Statistics of Random Processes, Springer-Verlag, New-York, 1978.

[18] Liptser, R. S. and Shiryaev, A. N. : Theory of Martingales, Kluwer Academic Publ., Dordrecht, 1989.

[19] Leland, W.E., Taqqu, M.S., Willinger, W. and Wilson, D.V. : On the selfsimilar nature of Ethernet traffic, I.E.E.E. / A.C.M. Trans. Networking, 2, (1), 1994, pp. 1-15.

[20] Lin, S.J., Stochastic analysis of fractional Brownian motions, Stochastics and Stochastics Reports, 55, 1995, pp. 121-140.

[21] Lundgren, T. and Chiang, D. : Solution of a class of singular integral equations, Quart. J. Appl. Math., 24, 1967, pp. 303-313.

[22] Lyons, T. : Differential equations driven by rough signals, I. An extension of an inequality of L.C. Young, Mathematical Research Letters, 1, (4), 1994, pp. 451-464.

[23] Mandelbrot, B.B. and Van Ness, J.W. : Fractional Brownian motions, fractional noises and applications, SIAM Review, 2, (10), 1968, pp. 422437. 
[24] Norros, I. : A storage model with self-similar input, Queueing Systems, 16, 1994, pp. 387-396.

[25] Norros, I. : On the use of the fractional Brownian motion in the theory of correctionless networks, I.E.E.E. Jour. on Sel. Ar. in Communications, 13, (6), 1995, pp. 953-962.

[26] Norros, I., Valkeila, E. and Virtamo, J. : An elementary approach to a Girsanov formula and other analytical results on fractional Brownian motions, Bernoulli, to appear, 1998.

[27] Samko, S.G., Kilbas, A.A. and Marichev, O.I. : Fractional Integrals and Derivatives, Gordon \& Breach Science, 1993.

[28] Samorodnitsky, G. and Taqqu, M.S. : Stable Non-Gaussian Random Processes: Stochastic Models with Infinite Variance, Chapman \& Hall, London, 1994.

[29] Zakai, M. : On the optimal filtering of diffusions processes, Zeit. Wahr. Verv. Geb., 11, 1969, pp. 230-243.

$\mathrm{RR} \mathrm{n}^{\circ} 3439$ 


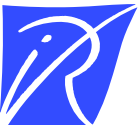

Unité de recherche INRIA Lorraine, Technopôle de Nancy-Brabois, Campus scientifique, 615 rue du Jardin Botanique, BP 101, 54600 VILLERS LÈS NANCY

Unité de recherche INRIA Rennes, Irisa, Campus universitaire de Beaulieu, 35042 RENNES Cedex Unité de recherche INRIA Rhône-Alpes, 655, avenue de l'Europe, 38330 MONTBONNOT ST MARTIN Unité de recherche INRIA Rocquencourt, Domaine de Voluceau, Rocquencourt, BP 105, 78153 LE CHESNAY Cedex Unité de recherche INRIA Sophia Antipolis, 2004 route des Lucioles, BP 93, 06902 SOPHIA ANTIPOLIS Cedex

Éditeur

INRIA, Domaine de Voluceau, Rocquencourt, BP 105, 78153 LE CHESNAY Cedex (France)

ISSN 0249-6399 